\title{
Comparison of segmentation of 2D and 3D EBSD measurements in polycrystalline materials
}

\author{
J. Staněk ${ }^{1 *}$, J. Kopeček ${ }^{2}$, P. Král ${ }^{3}$, I. Karafiátová ${ }^{4}$, F. Seitl ${ }^{4}$, V. Beneš ${ }^{4}$ \\ ${ }^{1}$ Department of Mathematics Education, Faculty of Mathematics and Physics, Charles University, \\ 18675 Prague, Czech Republic \\ ${ }^{2}$ Institute of Physics of the CAS, Department of Functional Materials, Na Slovance 2, 18200 Prague, Czech Republic \\ ${ }^{3}$ Institute of Physics of Materials, ASCR, Žižkova 22, 61662 Brno, Czech Republic \\ ${ }^{4}$ Department of Probability and Mathematical Statistics, Faculty of Mathematics and Physics, Charles University, \\ 18675 Prague, Czech Republic
}

Received 30 December 2019, received in revised form 26 April 2020, accepted 17 June 2020

\begin{abstract}
A comparison study of grain segmentation in $2 \mathrm{D}$ and $3 \mathrm{D}$ electron backscatter diffraction (EBSD) measurements of polycrystalline materials, based on a misorientation threshold, is presented. Using Laguerre tessellations with orientation marks, a simulation study that demonstrates a different sensitivity of segmentation in the case of low angle and high angle grain boundaries is involved. Then two metallic materials measured by 3D EBSD are investigated concerning segmentation, and there are given some recommendations for the misorientation thresholds. Computational tools such as DREAM 3D software enable significant data evaluation that is completed by a five-parameter analysis of grain boundaries.
\end{abstract}

Key words: electron backscatter diffraction (EBSD), grain boundaries, misorientation, tessellation

\section{Introduction}

The electron backscattered diffraction method (EBSD) has been a powerful tool in material science since the nineties, when a certain level of computerization and application of Hough transform took place, see $[1,2]$. Nowadays, we can perform not only 2D EBSD but exploiting focused ion beams (FIB), even 3D EBSD, by materials' slicing tomography [3]. The 3D EBSD data evaluation is a complicated process [4]. Still, we expect to be able to construct grains [5] and microstructure parameters by applying the same or similar procedures as in 2D EBSD data evaluation [6]. However, a discrepancy in the segmentation of data points into the grains in the $2 \mathrm{D}$ and $3 \mathrm{D}$ cases is apparent, since a nonconvex grain in 3D can be observed as more than one grain in a $2 \mathrm{D}$ slice.

The segmentation problem is deeply connected to the basic understanding of the polycrystals. Generally, the definition of grains as they were observed by founders of metallography was purely natural: They observed the boundaries - the separators, whereas grains or rather their interiors were homogeneous areas inside with defined or expected value of properties. Of course, it is known that a grain structure with the tessellation formula can be created [7]; nevertheless, EBSD is the final step reversing axiom to construct the grains [2]. We have a set of measured points in the EBSD method. We have positions of such points and their marks (e.g., Euler angles). Thus, here the interior is a quality, which exists, whereas the boundary has to be constructed. The easiest way how to create grains from the set of measured points is the following definition: the grain interior shall have the same or very similar properties. Thus, we construct grains by collecting the measured points with the defined property. The metallographic and EBSD grains must be then the same in the optimal case. However, the result of an experiment is not optimal. It contains some noise or even a kind of systematic aberrations, and the reconstruction of grain structure can be done only up to some level of accuracy.

*Corresponding author: tel.: +420 951553215; e-mail address: stanekj@karlin.m.cuni.cz 
The problem described above was omitted in computational materials science since the $3 \mathrm{D}$ data is frequently used in an elementary manner. Despite the explosion of three-dimensional methods in the last decades, the majority of 3D models use simple mathematical schemes to build the structure by a general procedure approaching the reality by just a few parameters, measured from $2 \mathrm{D}$ cuts of material [8].

Data obtained by 3D EBSD or 2D EBSD form a set of orientations of measured points in a $3 \mathrm{D}$ matrix (the points are called voxels in the sequel) or $2 \mathrm{D}$ matrix (pixels in the sequel), respectively. The EBSD method considers a grain to be the set of all points (voxels or pixels, respectively) with a similar orientation, which are surrounded by a boundary of a given misorientation threshold. The typical threshold in the EDAX OIM software is $5^{\circ}$ [9], but other choices are possible as well $[10,11]$. The obtained segmentation into the grains depends on the chosen misorientation threshold, of course, but even if we use a fixed misorientation threshold, we may get different results of the segmentation in $2 \mathrm{D}$ and $3 \mathrm{D}$, as shown in [12]. Therefore, the results obtained in 2D EBSD may not be easily compared with the results from $3 \mathrm{D}$ EBSD

In the paper, we present a comparison study of segmentation of $2 \mathrm{D}$ and $3 \mathrm{D}$ EBSD measurements in polycrystalline materials, based on a misorientation threshold. The main results of this topic are presented in Sections 2 and 3. The aim is to draw the reader's attention to the danger of obtaining misleading quantitative results about the geometry of microstructure when paying little attention to a proper segmentation. First, in Section 2, the problem is demonstrated in a simulation study. A generalized Laguerre tessellation modeling of polycrystalline microstructure measured by 3 D EBSD was used in [13]. We can simulate this model with a priori given distribution of misorientations. The influence of this distribution on the segmentation is shown.

In Section 3, we discuss the segmentation problem with two materials, namely the AlMgSc alloy after ECAP and annealing, and pure copper after highpressure torsion and annealing at room temperature. The polycrystalline microstructures are observed by 3D EBSD and 2D EBSD. The approaches presented in Section 2 for simulated toy data examples are discussed subsequently for both materials. The curve of dependence of the mean number of grains, in a single $2 \mathrm{D}$ slice, on the misorientation tolerance level, is plotted for both 2D and 3D methods. The differences are remarkable.

In Section 4, as an application to microstructure analysis of 3D EBSD data, we present a five-parameter analysis of grain boundaries in $\mathrm{Cu}$ specimen and suggest a physical interpretation. Three levels of segmentation are considered and discussed. The 3D mi- (a)
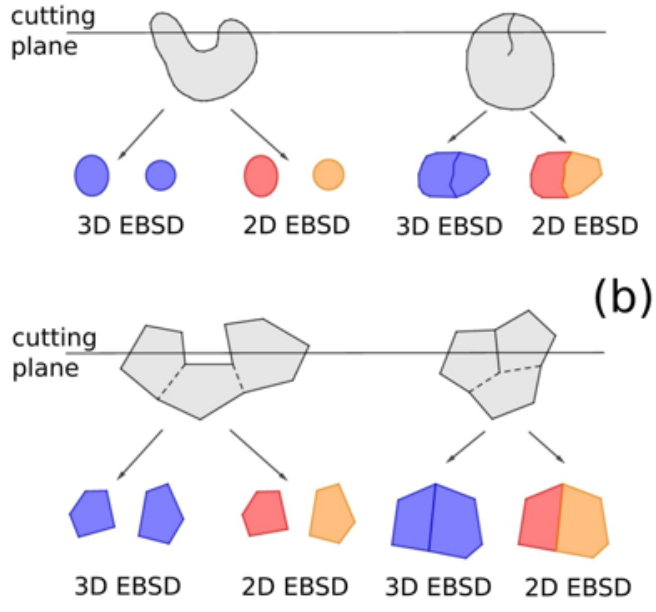

Fig. 1. Differences between slices processed from 2D EBSD and the same slice processed from 3D EBSD. The grey colour displays the real grains (in the plane, which is orthogonal to the cutting plane). The blue colour displays their $3 \mathrm{D}$ segmentation in the cutting plane, i.e., the parts are considered to belong to the same grain. Finally, the red and the orange colours show their $2 \mathrm{D}$ segmentation in which they are considered to be different grains. (a) The situation with a nonconvex grain or a grain with a boundary inside. (b) The situation with convex grains, boundaries with the misorientation smaller than the chosen threshold are denoted by the dashed line.

crostructure analyses make use of DREAM 3D software [14].

Some conclusions from Sections 2 and 3 are given in more detail in the final part of the paper.

\section{Differences between $2 \mathrm{D}$ and $3 \mathrm{D}$ segmentation}

The differences between segmentation into grains in $2 \mathrm{D}$ and $3 \mathrm{D}$ are caused by the fact that in a $2 \mathrm{D}$ cutting plane, two sets of voxels may be surrounded by a boundary defined by a given threshold of misorientation so that the sets are detected as two grains, while in 3D case, all these points belong to one larger set which is surrounded by a boundary. Therefore in the $3 \mathrm{D}$ case, all these points lie in the same grain, see Fig. 1. This behaviour can be observed when the grains are nonconvex, or when there is a boundary of the given misorientation inside the grain. One could conclude such a situation to be unrealistic, but the same behaviour can be observed in materials formed by convex grains with very low local misorientation (all voxels from a single grain have nearly the same orientation), if the chosen misorientation threshold is higher than the minimal misorientation of the neigh- 

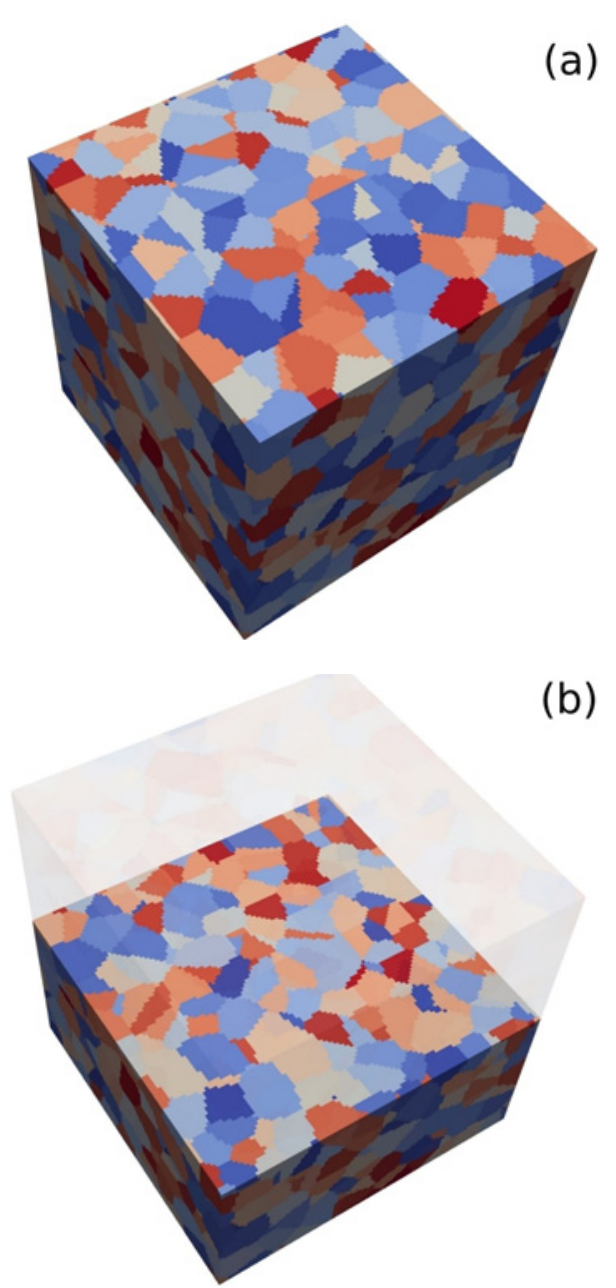

Fig. 2. A Laguerre tessellation simulated from the 3D stochastic Gibbs-Laguerre model in a cubic window. The pattern (a) involves 1560 grains coloured at random. In (b), there is a $2 \mathrm{D}$ slice taken from the middle of the pattern (a).

bouring grains. In this case, the segmentation joins more real grains into one larger grain. The situation is visualized in Fig. 1b. In both cases in the figure, we have three grains which are segmented in 3D into one grain, because some boundaries (dashed lines) have lower misorientation than the chosen threshold, but the segmentation in $2 \mathrm{D}$ (in the cutting plane) separates these grains because the boundary in the cutting plane (marked by the solid line) has greater misorientation than the chosen threshold.

In this section, we study the difference between segmentation into grains in $2 \mathrm{D}$ and $3 \mathrm{D}$ for simulated data. As a model for a polycrystalline microstructure, we use the Laguerre tessellation [15], see Fig. 2. The Markov chain Monte Carlo method of simulation of a Gibbs-Laguerre tessellation [16] is extended by adding the Euler angles to the state space. For this model of a tessellation structure, two different scenarios of orien-
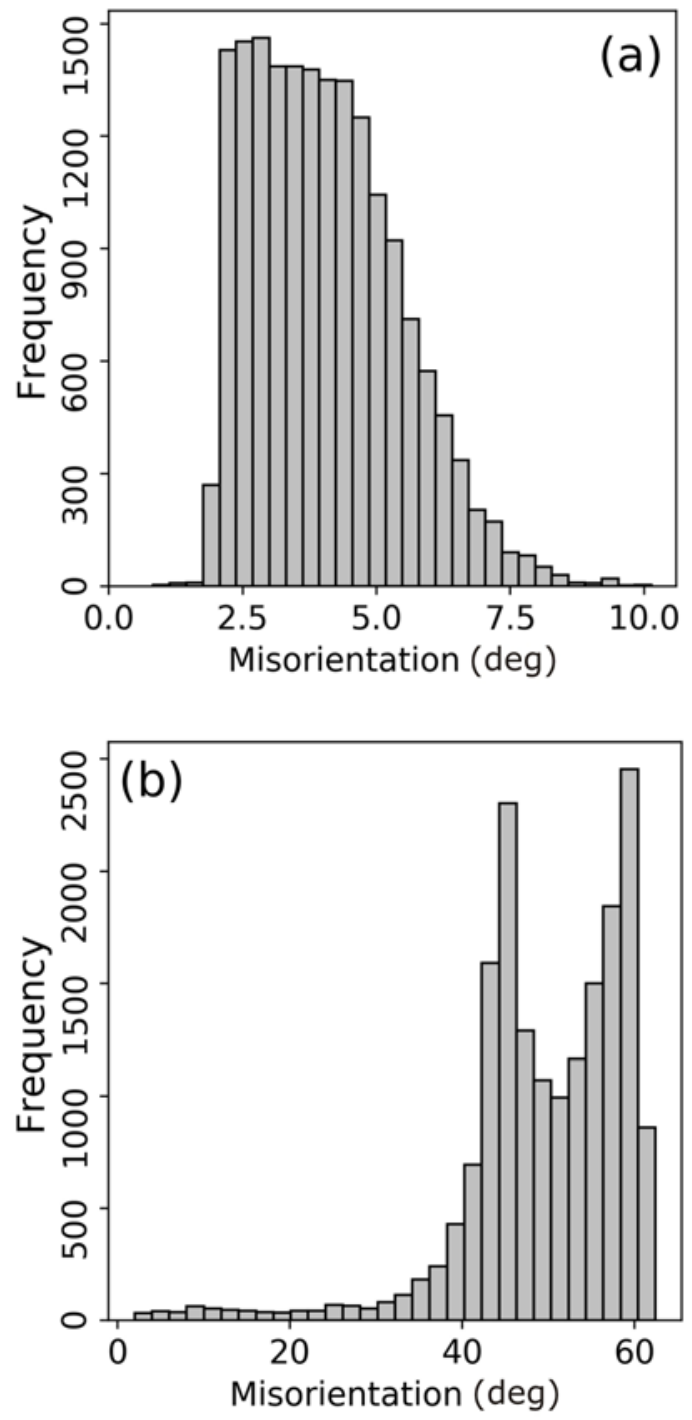

Fig. 3. Histograms of misorientations between neighbouring grains in the simulated data. (a) the case (A) - low angle grain boundaries, (b) the case (B) - high angle grain boundaries.

tations of cubic crystal grid of grains were simulated, namely:

(A) the misorientation between neighbouring grains is low, (see Fig. 3a),

(B) the misorientation between neighbouring grains is high, (see Fig. 3b).

These two cases represent the situations, when the material prefers low angle grain boundaries (LAGB) or high angle grain boundaries (HAGB), respectively. For further purposes, we work with voxelized data $(100 \times 100 \times 100$ voxels $)$, where each voxel has its orientation given by the associated grain. The data are processed by the software DREAM.3D. Since the cells in the Laguerre tessellation (representing grains in our case) are convex, and all voxels in each grain are assigned the same orientation, the simulated data 

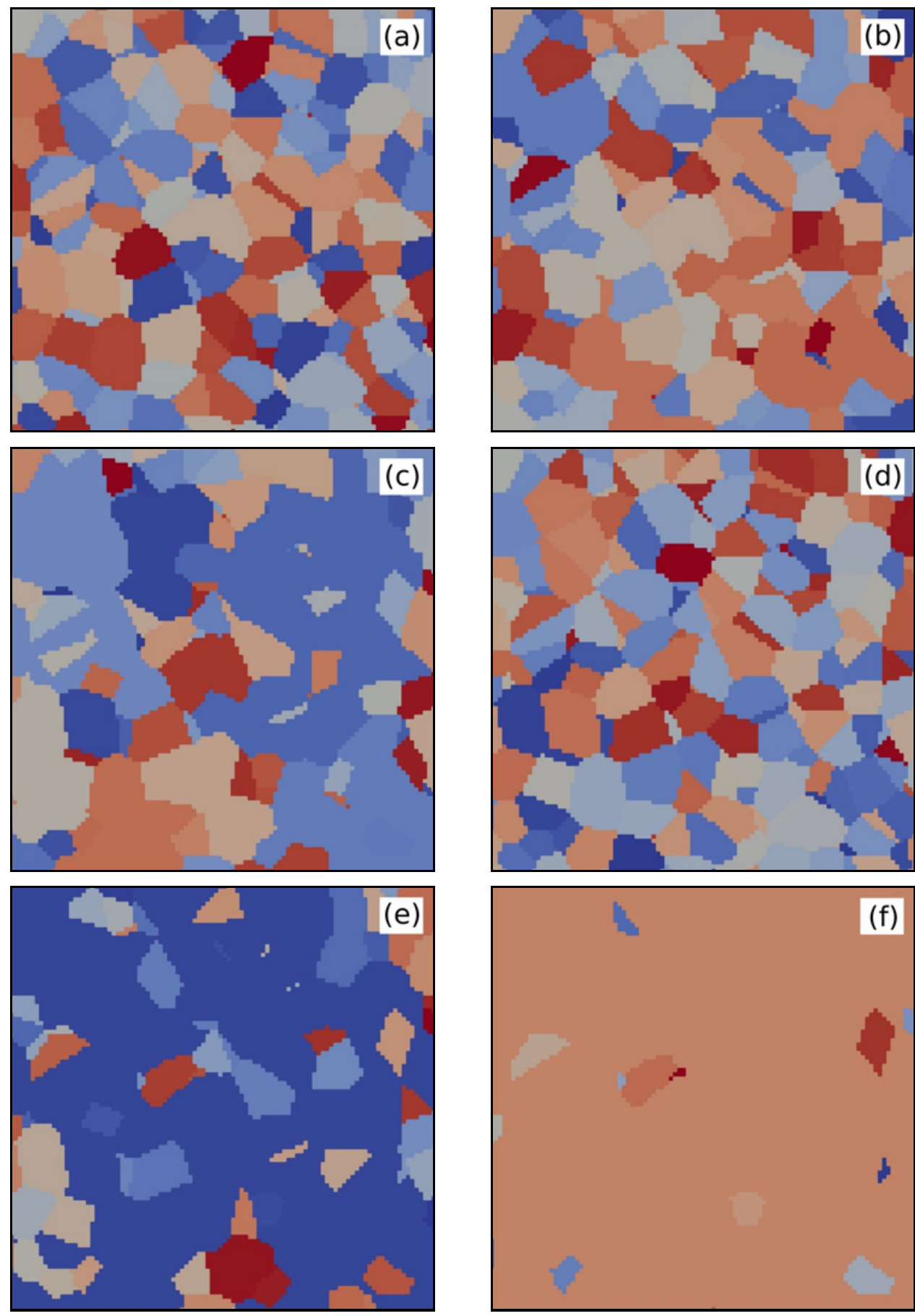

Fig. 4: The segmentation of simulated data for the case (A) in 2D with the misorientation threshold (a) $2^{\circ}$, (b) $2.5^{\circ}$, and (c) $3^{\circ}$ and the same section from $3 \mathrm{D}$ reconstruction with the misorientation threshold (d) $2^{\circ}$, (e) $2.5^{\circ}$, and (f) $3^{\circ}$. The grains are coloured at random.

represent a material without noise, i.e., all the grains are well defined.

To compare the results obtained in $2 \mathrm{D}$ and $3 \mathrm{D}$, the filter 'Segment Features (Misorientation)' with a chosen misorientation threshold for the entire 3D data set is used first. Then, a single slice from the processed $3 \mathrm{D}$ data is cut out. This slice is treated separately, i.e., only the voxels from the slice for the segmentation into grains are considered, using the same filters and the same settings as in 3D. In this way, two different segmentations of the same slice are received, which are comparable. The first one represents the processing in $3 \mathrm{D}$, while the second one represents the processing in $2 \mathrm{D}$.

Just note that in the present study, the slice number 50 is processed, which is shown in Fig. $2 \mathrm{~b}$. 

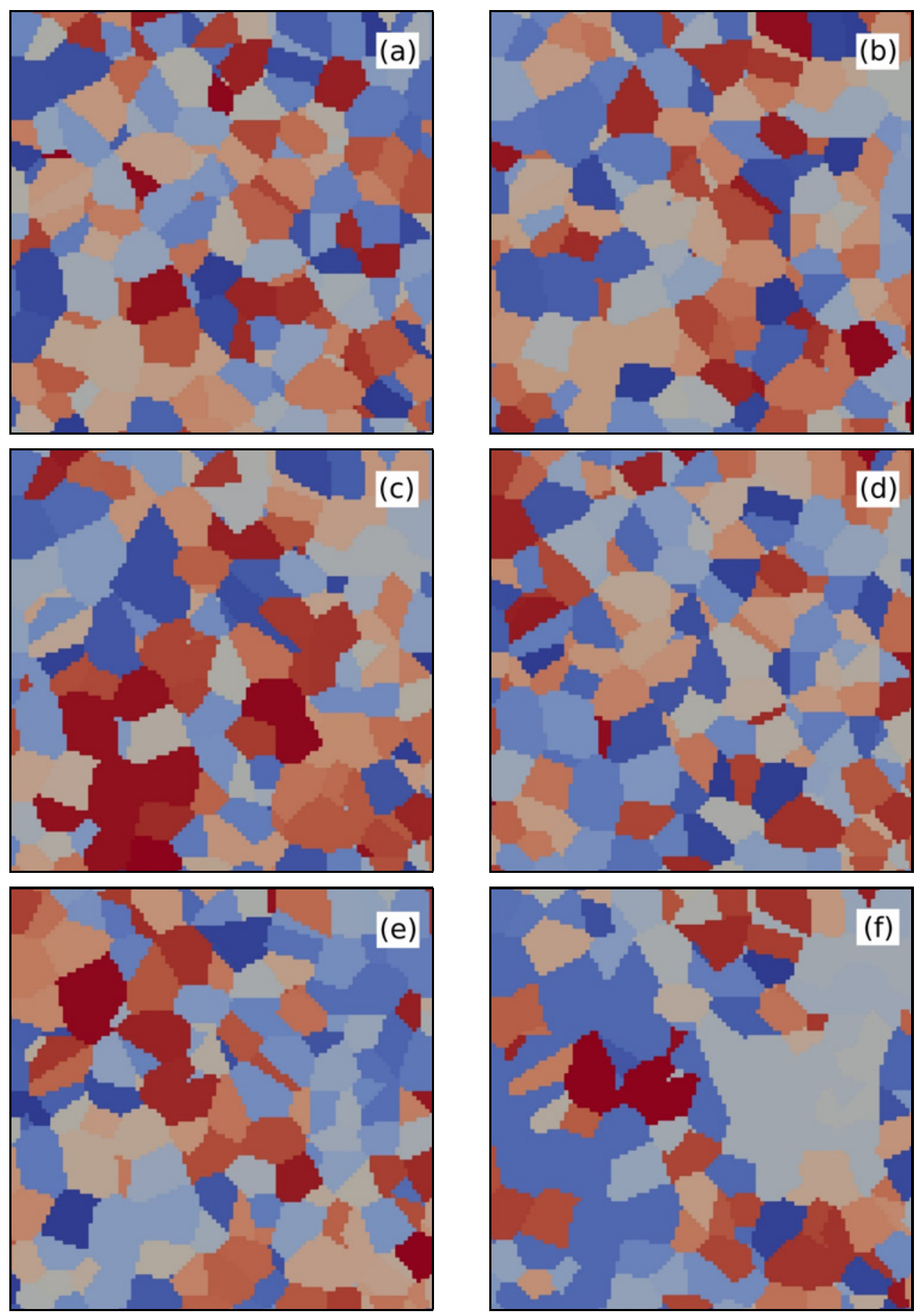

Fig. 5. The segmentation of simulated data for the case (B) in $2 \mathrm{D}$ with the misorientation threshold (a) $2^{\circ}$, (b) $36^{\circ}$, and (c) $40^{\circ}$ and the same section from $3 \mathrm{D}$ reconstruction with the misorientation threshold (d) $2^{\circ}$, (e) $36^{\circ}$, and (f) $40^{\circ}$. The grains are coloured at random.

The difference between segmentation in 2D and 3D in the case (A) is shown in Fig. 4 . In this case, the misorientation thresholds equal to $2^{\circ}, 2.5^{\circ}$, and $3^{\circ}$ are used. Note that $0.15,13.2$, and $26.5 \%$ of boundaries have misorientation lower than $2^{\circ}, 2.5^{\circ}$, and $3^{\circ}$, respectively. While for the threshold of $2^{\circ}$, we get nearly the same segmentation in both cases, for the thresholds of $2.5^{\circ}$ and $3^{\circ}$, the segmentations are entirely different.
For the case (B), a similar result is shown in Fig. 5. In this case, we use the misorientation thresholds equal to $2^{\circ}, 35^{\circ}$, and $40^{\circ}$. Note that $0.01,5.0$, and $9.4 \%$ of boundaries have misorientation lower than $2^{\circ}, 35^{\circ}$, and $40^{\circ}$, respectively. While we get similar segmentations for all chosen thresholds between $2^{\circ}$ and $35^{\circ}$, the difference between segmentations for the threshold of $40^{\circ}$ is not marginal. 

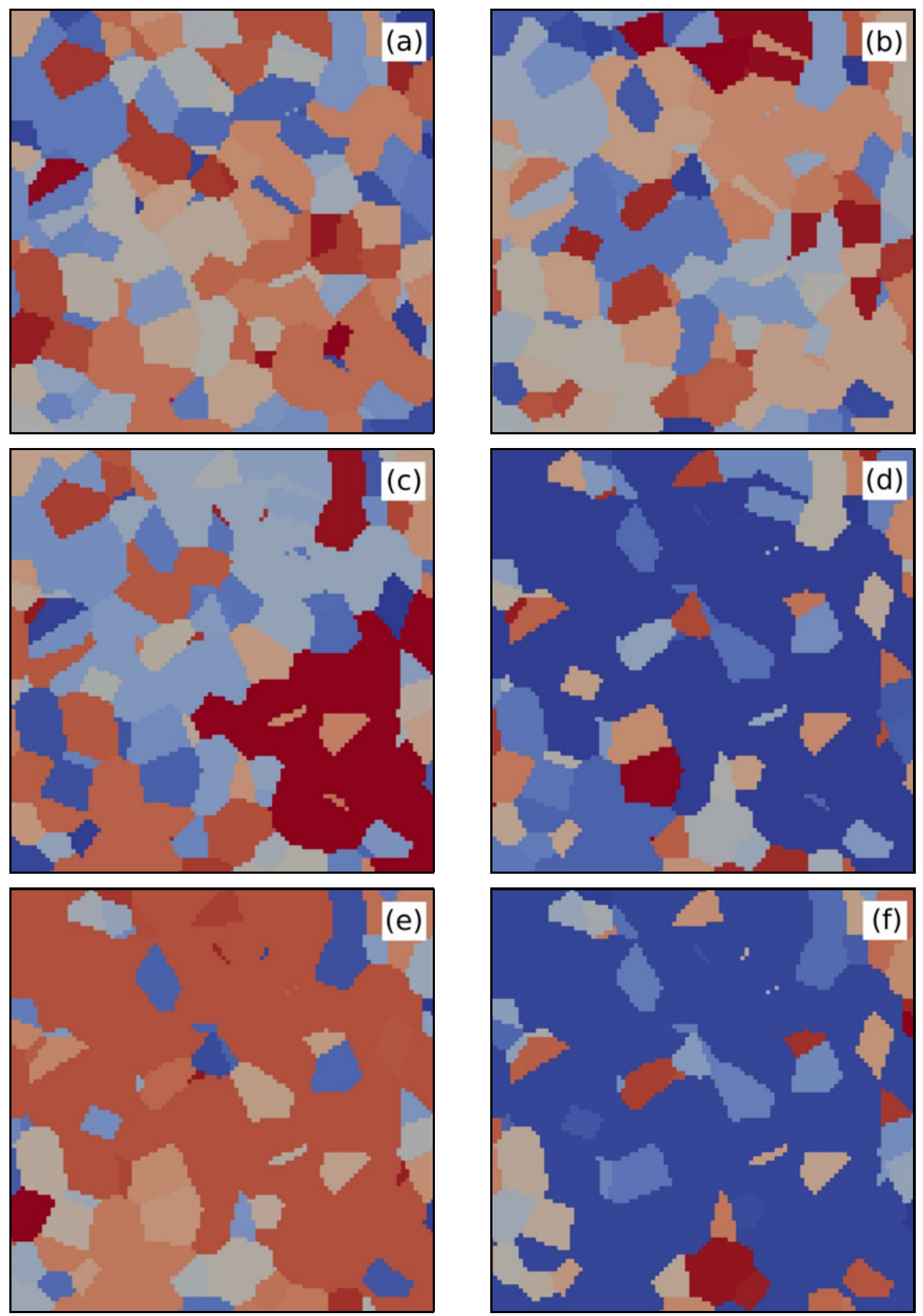

Fig. 6. The segmentation of simulated data with the chosen misorientation threshold $2.5^{\circ}$ for (a) $2 \mathrm{D}$ data, (b) $3 \mathrm{D}$ data 10 slices, (c) 3D data - 20 slices, (d) 3D data - 30 slices, (e) 3D data - 50 slices, and (f) 3D data - 100 slices. The grains are coloured at random.

The question is, how does the difference between segmentation in 2D and 3D depend on the thickness of the specimen. Obviously, if we work with a 3D data set which contains only a few slices, the difference between segmentation in 2D and 3D should be marginal, since there is not enough space for the effect described in Fig. 1. To study this dependence, the simulated data (A) were used, and the focus was given on the slice number 50, which has already been studied. Six scenarios were compared: (a) segmentation in 2D, where only the slice number 50 was used, (b) segmentation in $3 \mathrm{D}$ with the data set of 10 slices enumerated $45-54$, (c) segmentation in $3 \mathrm{D}$ with the data set of 20 slices enumerated 40-59, (d) segmentation in 3D with the data set of 30 slices enumerated 35-64, (e) segmentation in $3 \mathrm{D}$ with the data set of 50 slices enumer- 

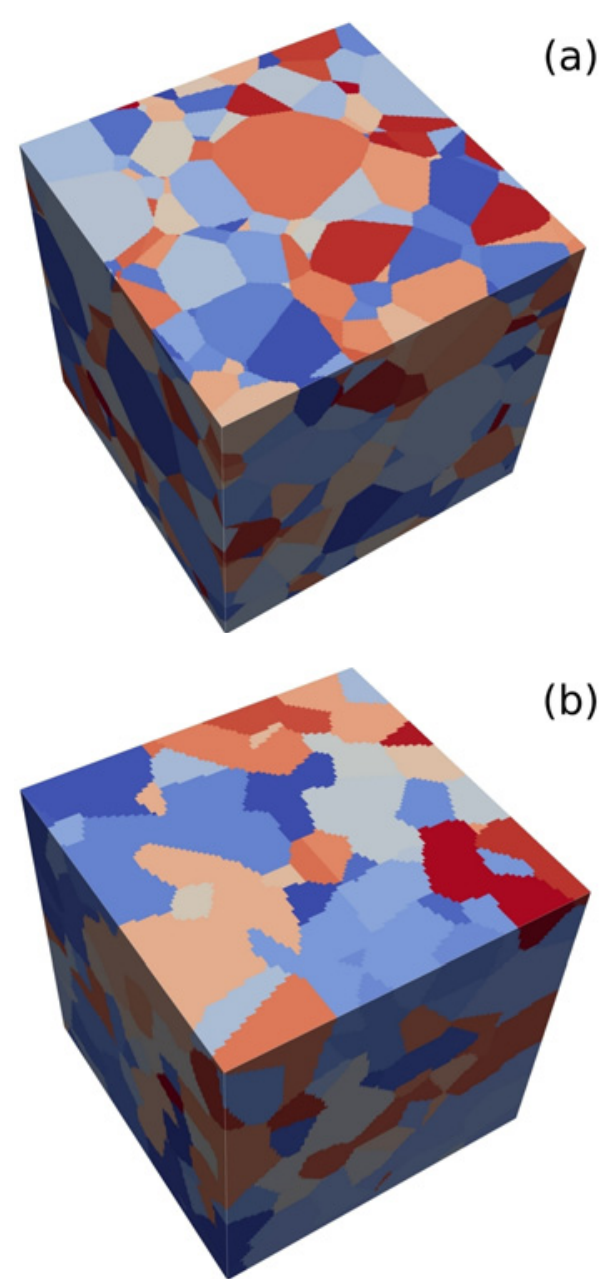

Fig. 7. The simulated data: (a) case (C) and (b) case (D).

ated 25-74, and (f) segmentation in 3D with the entire data set (100 slices). The result for the misorientation threshold $2.5^{\circ}$ is shown in Fig. 6 . One can see that the difference between the segmentation in $2 \mathrm{D}$ and $3 \mathrm{D}$ is marginal when the specimen is thin (10 slices in our case, i.e., $100 \times 100 \times 10$ voxels), on the other hand, the difference is significant when the specimen has 30 or more slices. The results are very similar if the data set with 50 slices (e) or the entire data set with 100 slices (f) is used.

Another question is whether the grain size and morphology affect the difference between the segmentation in $2 \mathrm{D}$ and $3 \mathrm{D}$ as well. To investigate this dependence, two more simulated data sets were added to our study, namely (C) the Laguerre tessellation with a few distinctively larger grains and (D) the tessellation with nonconvex grains, see Fig. 7. The orientation of the crystals in the cases (C) and (D) is chosen in a similar way as in the case (A); therefore, the misorientation between neighbouring grains is low, and the histograms of the misorientation are similar to the histogram in Fig. 3a. The segmenta- tion in $2 \mathrm{D}$ and $3 \mathrm{D}$ for the cases $(\mathrm{A}),(\mathrm{C})$, and $(\mathrm{D})$ is investigated. For all three cases, the segmentation with three misorientation thresholds is studied. The first one is $0.1^{\circ}$ (called $0 \%$ misorientation segmentation in the sequel), the second one is chosen so that $10 \%$ of boundaries have lower misorientation (called $10 \%$ misorientation segmentation in the sequel). The last one corresponds to $15 \%$ of boundaries with misorientation lower than the chosen threshold (called 15\% misorientation segmentation in the sequel). The segmentation in $3 \mathrm{D}$ with such thresholds is shown in Fig. 8. The behaviour for all three cases (A), (C), and (D) is very similar. The $10 \%$ misorientation segmentation contains larger grains, which are not presented in $0 \%$ misorientation segmentation, and all three $15 \%$ misorientation segmentations contain a single giant grain that covers most of the area of the slice. Note that the slice studied in the case $(\mathrm{A})$ is the slice number 50, which has already been considered above. Note that the results in 2D segmentation are similar for all three cases, i.e., the behaviour is similar to that one presented in Figs. $4 a-c$. Therefore the segmentation in $2 \mathrm{D}$ is not visualized in this paper.

Note that similar results are obtained when processing the other slices in the data set. Thus, based on this short simulation study, it can be concluded that the difference between processing of the data in $2 \mathrm{D}$ and $3 \mathrm{D}$ is significant if at least $10-15 \%$ of boundaries have misorientation lower than the threshold chosen for the segmentation procedure. The difference between the processing of the data in $2 \mathrm{D}$ and $3 \mathrm{D}$ is significantly affected by the thickness of the sample, but it seems that the morphology of the grains does not play an important part.

\section{Real data}

After the analysis of the simulated data in the previous section, two real data sets are investigated, and the segmentation into the grains in $2 \mathrm{D}$ and $3 \mathrm{D}$ is compared. The materials are described below in Sections 3.1. and 3.2. It has to be added that most microstructures show a particular orientation spread within the grain interiors, and this is the result of the processing history. It should be distinguished from acquisition noise, although for grain segmentation purposes, they are usually not separated. Both materials have been measured by the 3DEBSD method, which provides the data in the form of $2 \mathrm{D}$ slices. Such slices can be subsequently joined into a $3 \mathrm{D}$ image. Processing of the data, such as removing noise, matching $3 \mathrm{D}$ slices into a 3D image or removing grains, which are too small, etc., has been made by the software DREAM.3D. 2D and $3 \mathrm{D}$ are compared in the same way as in the previous section. First, a $2 \mathrm{D}$ slice processed separately 

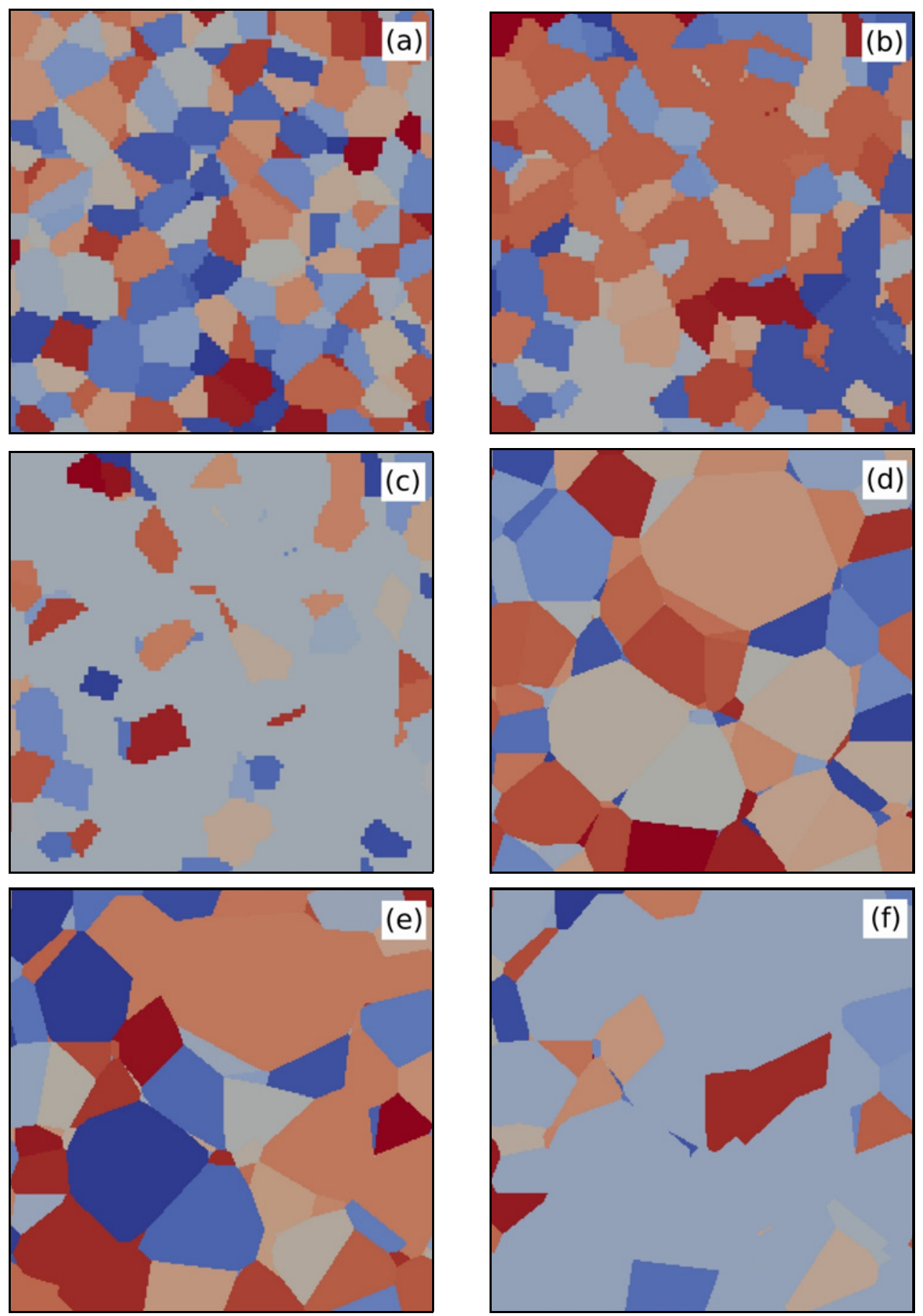

Fig. 8a-f. The segmentation of the simulated data in 3D for the case (A) with the misorientation threshold (a) $0.1^{\circ}$, (b) $2.39^{\circ}$, and (c) $2.58^{\circ}$. The segmentation of the simulated data in $3 \mathrm{D}$ for the case (C) with the misorientation threshold (d) $0.1^{\circ}$, (e) $2.22^{\circ}$, and (f) $2.34^{\circ}$.

by DREAM.3D is chosen, and the obtained result is quite similar to the results from working with $2 \mathrm{D}$ data. Then, the entire $3 \mathrm{D}$ data is processed in the same way and after $3 \mathrm{D}$ reconstruction, the chosen slice is cut out. In this way, two processings of the same slice are obtained (one in 2D and one in $3 \mathrm{D}$ ), which are comparable.

\subsection{Data of AlMgSc alloy}

The first investigated material is the aluminium alloy $\mathrm{Al}-3 \mathrm{Mg}-0.2 \mathrm{Sc}$, which is an alloy known for its superplasticity at high deformation rates [17]. The sample was subjected to severe plastic deformation by equal channel angular pressing (ECAP), annealed 

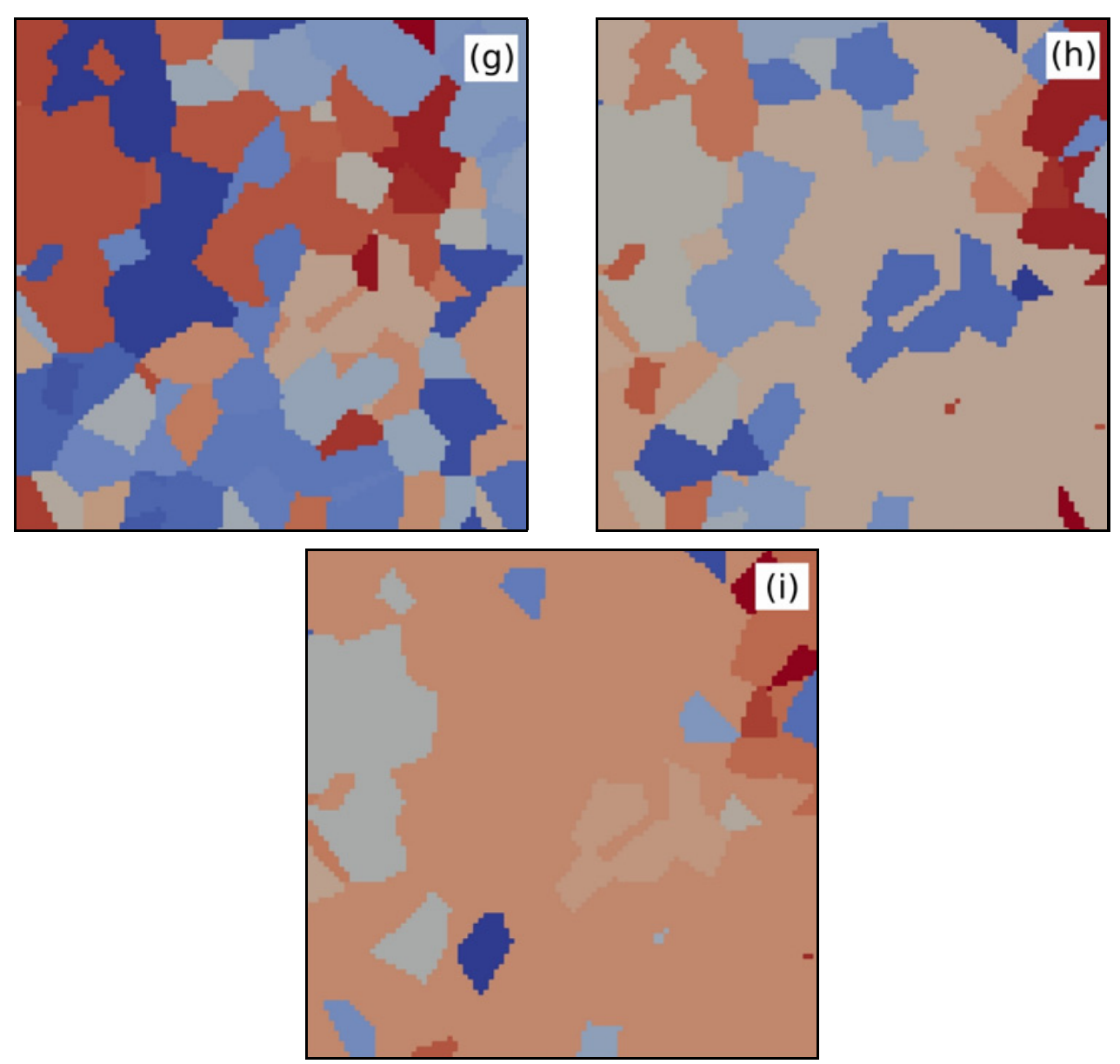

Fig. $8 \mathrm{~g}-\mathrm{i}$. The segmentation of the simulated data in $3 \mathrm{D}$ for the case (D) with the misorientation threshold $(\mathrm{g}) 0.1^{\circ},(\mathrm{h})$ $2.07^{\circ}$, and (i) $2.30^{\circ}$.

subsequently for $1 \mathrm{~h}$ at $400^{\circ} \mathrm{C}$ and investigated by three-dimensional electron backscattered diffraction (3D-EBSD). Note that the size of the specimen was $98.4 \times 71.7 \times 35.1 \mu^{3}$ and it has been already investigated in detail in [12], where the difference between detection of grains in 2D and 3D is also briefly discussed. In the present paper, this difference is focused on more details. Since the sample includes a lot of noise, filters in DREAM.3D have to be used to minimize the effect of the noise. The data as processed in DREAM.3D, where the grains are detected by using the filter 'Segment Features (Misorientation)' with a misorientation threshold of $2^{\circ}$, are visualized in Fig. 9. Note that the choice of misorientation threshold as well as the settings for the procedure of removing noise firmly influence the number of detected grains. In our case, the procedure detects 74579 grains, but if we do not remove the noise and small grains (namely the grains consisting of less than 8 voxels), the procedure detects more than one million grains, where most of them are very small (few voxels only).

Firstly, we compare the mean number of grains in a slice with the misorientation threshold chosen for the segmentation procedure. The number of small grains

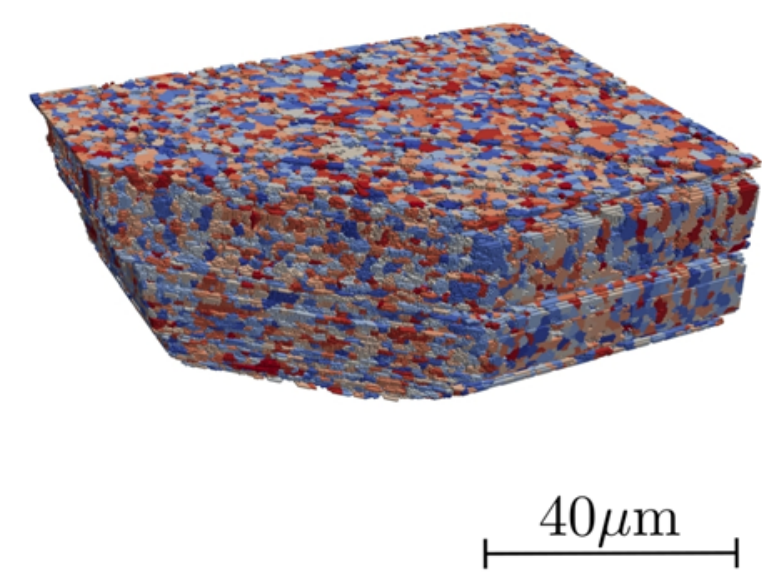

Fig. 9. The visualization of $3 \mathrm{D}$ data of AlMgSc alloy excluding the noisy part of the specimen as well as very small grains. The remaining part involves 74579 grains.

in a single slice is strongly affected by the noise, and unfortunately, it is not clear how to remove this effect in the same way for both dimensions. Therefore, we focus only on the mean number of the largest grains, 

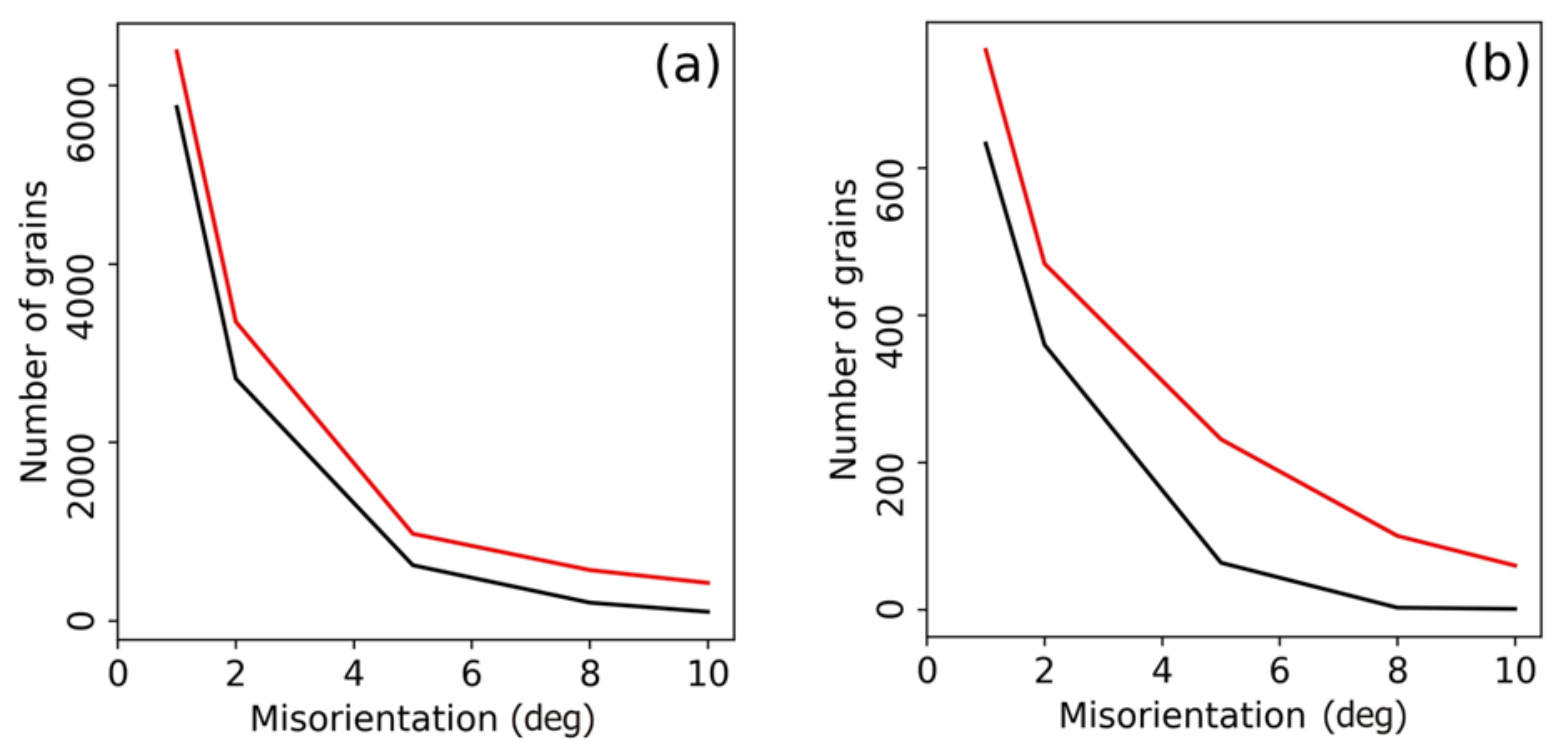

Fig. 10. Data of AlMgSc alloy. Dependence of the mean number of grains in a single slice on the misorientation threshold while processing by 2D EBSD method (red line) and 3D EBSD method (black line) for (a) the largest grains which cover in total $90 \%$ of the whole slice and (b) the largest grains which cover in total $50 \%$ of the whole slice (right).

which cover more than 50 or $90 \%$, respectively, of the area of the entire slice, see Fig. 10. In this way, the influence of the noise is eliminated, and the results are comparable. The difference in segmentation into grains is significant, mainly when we focus on the mean number of grains, which cover more than $50 \%$ of the area, and the chosen misorientation threshold is larger $\left(5^{\circ}, 8^{\circ}\right.$, or $\left.10^{\circ}\right)$. For example, for the misorientation threshold of $8^{\circ}$ and $10^{\circ}$, the mean number of grains covering more than one half of the slice is 2.66 and 1 , respectively, in $3 \mathrm{D}$, but it is 100.17 and 59.83 , respectively, in the two-dimensional case. The percentage of grain boundaries with misorientation lower than $5^{\circ}, 8^{\circ}$, and $10^{\circ}$ is $7.77,18.74$, and $25.64 \%$, respectively. Similarly, as in the previous section, we observe that when the percentage of grain boundaries, which has the misorientation lower than a chosen threshold, is more than $10 \%$, the difference between segmentation into grains is $2 \mathrm{D}$, and $3 \mathrm{D}$ is significant. In the real data sets, we can also see the difference in segmentation for a small misorientation threshold, which was not observed in simulated data. It is caused by the fact that the segmentation in $2 \mathrm{D}$ is more sensitive to the noise. This phenomenon will be shown in the next section.

In general, the segmentation into grains in $2 \mathrm{D}$ detects more grains than in the $3 \mathrm{D}$ case. Even if the numbers of grains are similar, the 3D segmentation tends to detect a few large grains, which may not be presented in the 2D segmentation. Such difference may not be visible in histograms or mean values, because if there are many grains in both cases, few large grains in 3D segmentation cannot change histograms or mean values of observed characteristics significantly. Let us
Ta ble 1. Data of AlMgSc alloy; mean and standard deviation (SD) of the area of grains and of the number of neighbours of grains for segmentation in $2 \mathrm{D}$ and $3 \mathrm{D}$

\begin{tabular}{lcc}
\hline & Area $\left(\mu \mathrm{m}^{2}\right)$ & Number of neighbours \\
\hline Mean (2D) & 1.250 & 4.627 \\
SD (2D) & 2.61 & 3.62 \\
Mean (3D) & 1.376 & 4.735 \\
SD (3D) & 13.80 & 18.09 \\
\hline
\end{tabular}

demonstrate it by our data set. We chose the misorientation threshold equal to $5^{\circ}$, which is the threshold usually chosen in the EDAX OIM software. Figure 11 shows the histograms of sphericity, which is a commonly used shape characteristic, see [18]. The whole histograms in Figs. 11a,b seem to be very similar. A difference can be observed in their cuts Figs. 11c,d. Here, it is seen that in the histogram Fig. 11c for the $3 \mathrm{D}$ case, there are a few grains with the sphericity lower than $0: 3$, while such grains are not present in the histogram Fig. 11d for 2D. This is caused by the fact that only the largest grains that are present just in 3D segmentation have such small sphericity. Similarly, in Table 1, we observe that mean values of the area or the number of neighbours of grain do not differ much in 2D and 3D segmentation, while the difference is clearly visible when we compare standard deviations of these characteristics. Analogously we compared the number of the largest grains covering the given percentage of the area, see Table 2, or the image of segmentation of one slice, see Fig. 12. 

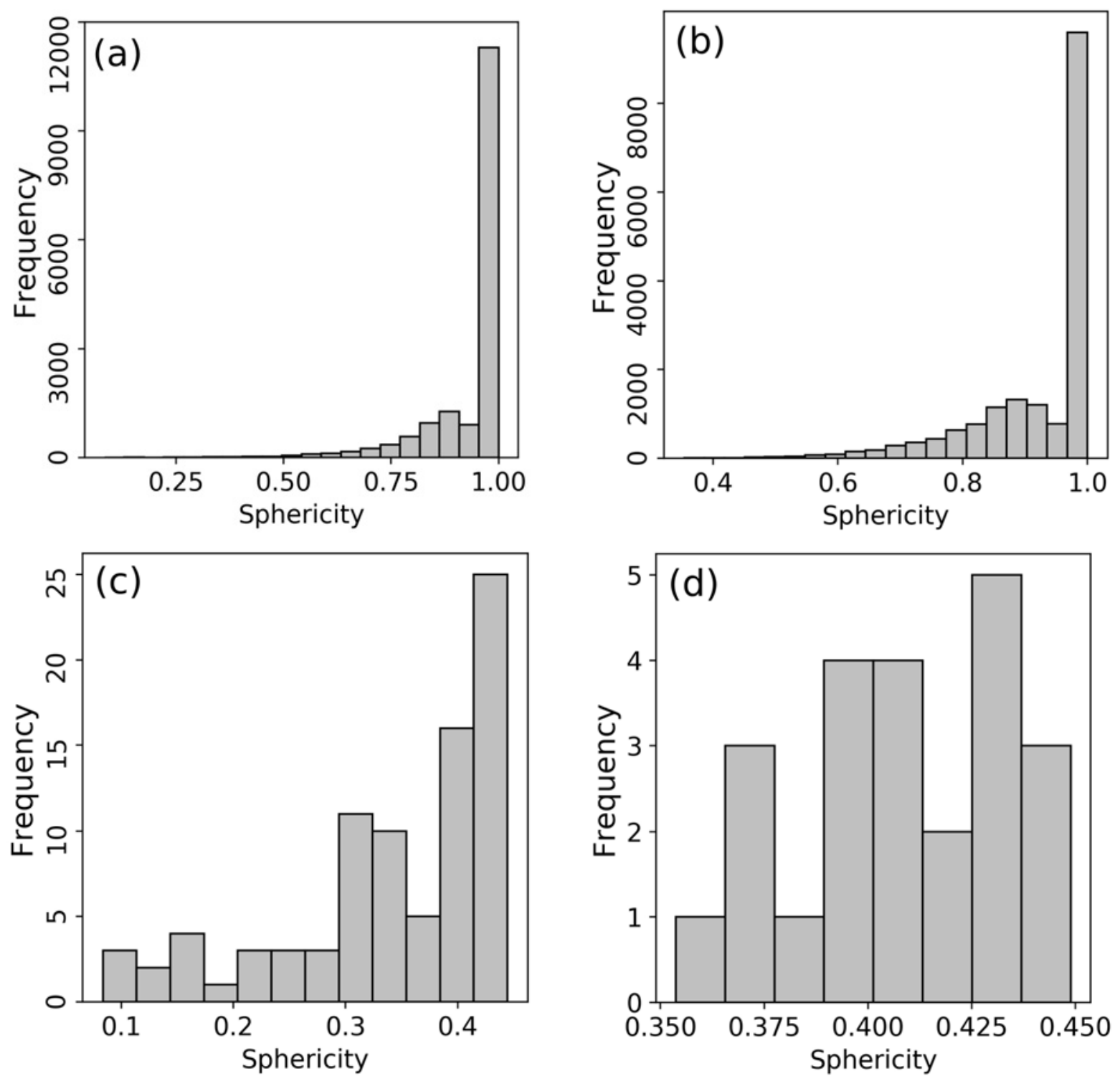

Fig. 11. Data of AlMgSc alloy. Histograms of the sphericity of grains with the misorientation tolerance of $5^{\circ}:$ (a) all grains in 3D, (b) all grains in 2D, (c) grains with sphericity lower than 0:45 in 3D, and (d) grains with sphericity lower than $0: 45$ in $2 \mathrm{D}$.

Ta b l e 2. Data of AlMgSc alloy; the number of the largest grains covering a given percentage of the area

\begin{tabular}{rrrrrc}
\hline & $100 \%$ & $75 \%$ & $50 \%$ & $25 \%$ & $10 \%$ \\
\hline $3 \mathrm{D}$ & 16137 & 1648 & 368 & 18 & 2 \\
$2 \mathrm{D}$ & 17119 & 3287 & 1378 & 404 & 94 \\
\hline
\end{tabular}

\subsection{Ultrafine-grained copper}

The second material, investigated in this section, is an ultrafine-grained copper. The dimensions of investigated microstructure (13) were $X=18.88 \mu \mathrm{m}, Y=$ $14.00 \mu \mathrm{m}$, and $Z=5.88 \mu \mathrm{m}$. The $X$-direction is paral- lel to the radius of HPT disk, $Y$-direction is parallel to the torsion axis of the HPT disk, and $Z$-direction corresponds to the HPT shear direction. The specimen was processed by 10 revolutions of high-pressure torsion and subsequently annealed at room temperature for 6 years. Note that the sample has already been in detail investigated in [19], but the difference between the detection of grains in $2 \mathrm{D}$ and $3 \mathrm{D}$ was not studied there. On the contrary to the aluminium alloy, this specimen has a small local misorientation (the majority of the area exhibits the local misorientation up to $0.5^{\circ}$ ), and it does not contain a lot of noise. Moreover, $90 \%$ of grain boundaries has the misorientation larger than $15^{\circ}$; thus, we obtain similar segmentation for the thresholds $1^{\circ}, 2^{\circ}, 5^{\circ}$, or $10^{\circ}$.

The data processed by DREAM.3D are shown in 

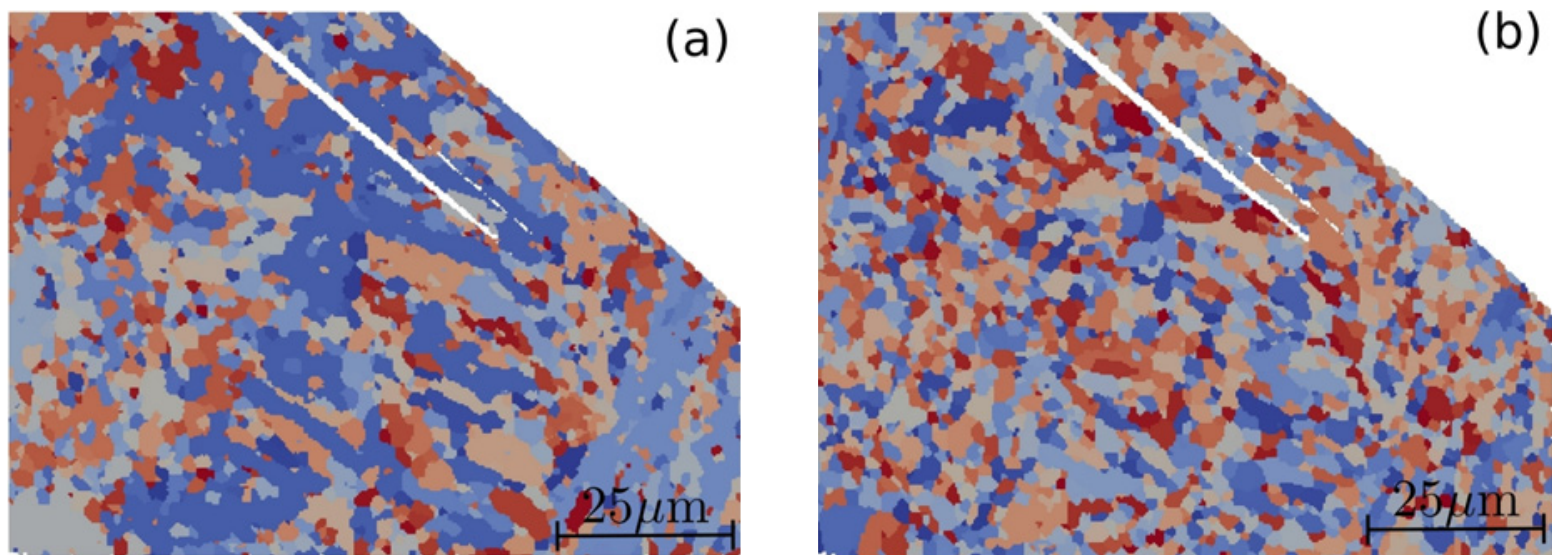

Fig. 12. Data of AlMgSc alloy. Segmentation of a sampled slice (namely the slice No. 102), the misorientation threshold is equal to $5^{\circ}$ : (a) segmentation in 3D (1412 grains), and (b) segmentation in 2D (2149 grains).

(a)

(b)

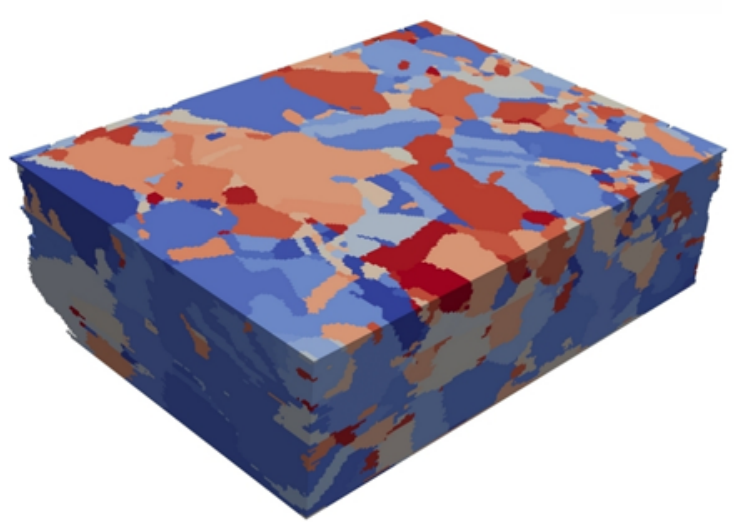

$\stackrel{5 \mu \mathrm{m}}{\longmapsto}$

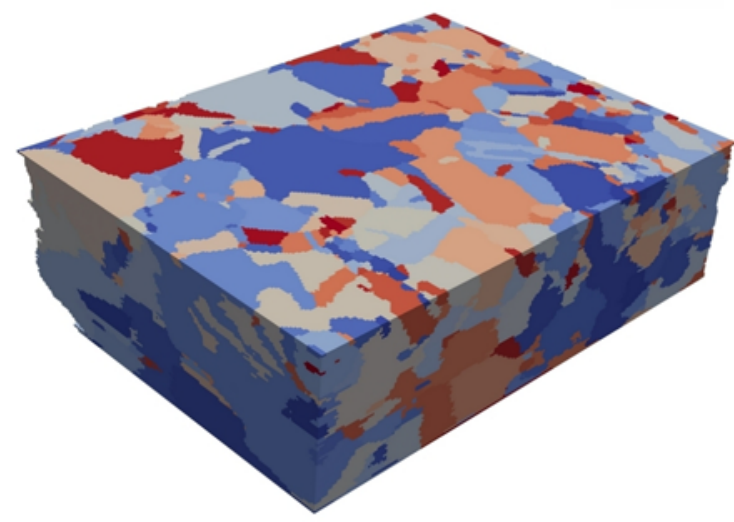

$5 \mu \mathrm{m}$

Fig. 13. 3D microstructure of pure copper data (grains coloured at random), (a) the segmentation with the misorientation tolerance of $1^{\circ}$, and (b) the segmentation with the misorientation tolerance of $5^{\circ}$.

Fig. 13. Note that two different misorientation thresholds, namely $1^{\circ}$ and $5^{\circ}$, are used, but the obtained segmentations are very similar. The differences between the segmentation in $2 \mathrm{D}$ and the segmentation in $3 \mathrm{D}$ with a different choice of the misorientation threshold are shown in Fig. 14. The results are similar in both cases, but the $2 \mathrm{D}$ segmentation is more sensitive to noise (only $3.46 \%$ of grain boundaries have misorientation lower than $5^{\circ}$ ).

If we compare the mean number of the largest grains that cover more than 50 or $90 \%$, respectively, of the area of the entire slice, the difference between $2 \mathrm{D}$ and $3 \mathrm{D}$ case is observable, see Fig. 15. It is caused by two facts. Firstly, as mentioned above, the 2D segmentation is more sensitive to noise (this is significant for a small segmentation threshold); therefore, it contains more tiny grains than in the 3D case. Secondly, the grains are not convex, therefore in the 2D case, more parts of a real grain segmented into more grains can be observed. In the $3 \mathrm{D}$ case, these parts belong to a single grain, and, therefore, all these parts are segmented into one grain (see Fig. 1).

On the other hand, the difference is negligible when comparing the characteristics like the area of grains, the sphericity of grains, or the number of grain neighbours, see Fig. 16.

In this case, the segmentation for a larger misorientation threshold is compared, for example, $25^{\circ}$, see Fig. 17, the difference is significant, and we get a similar result to the previous section more precisely, $18 \%$ of grain boundaries have the misorientation lower than $25^{\circ}$.

By deduction, we can conclude Section 3 similarly as in the previous section that the percentage of the 

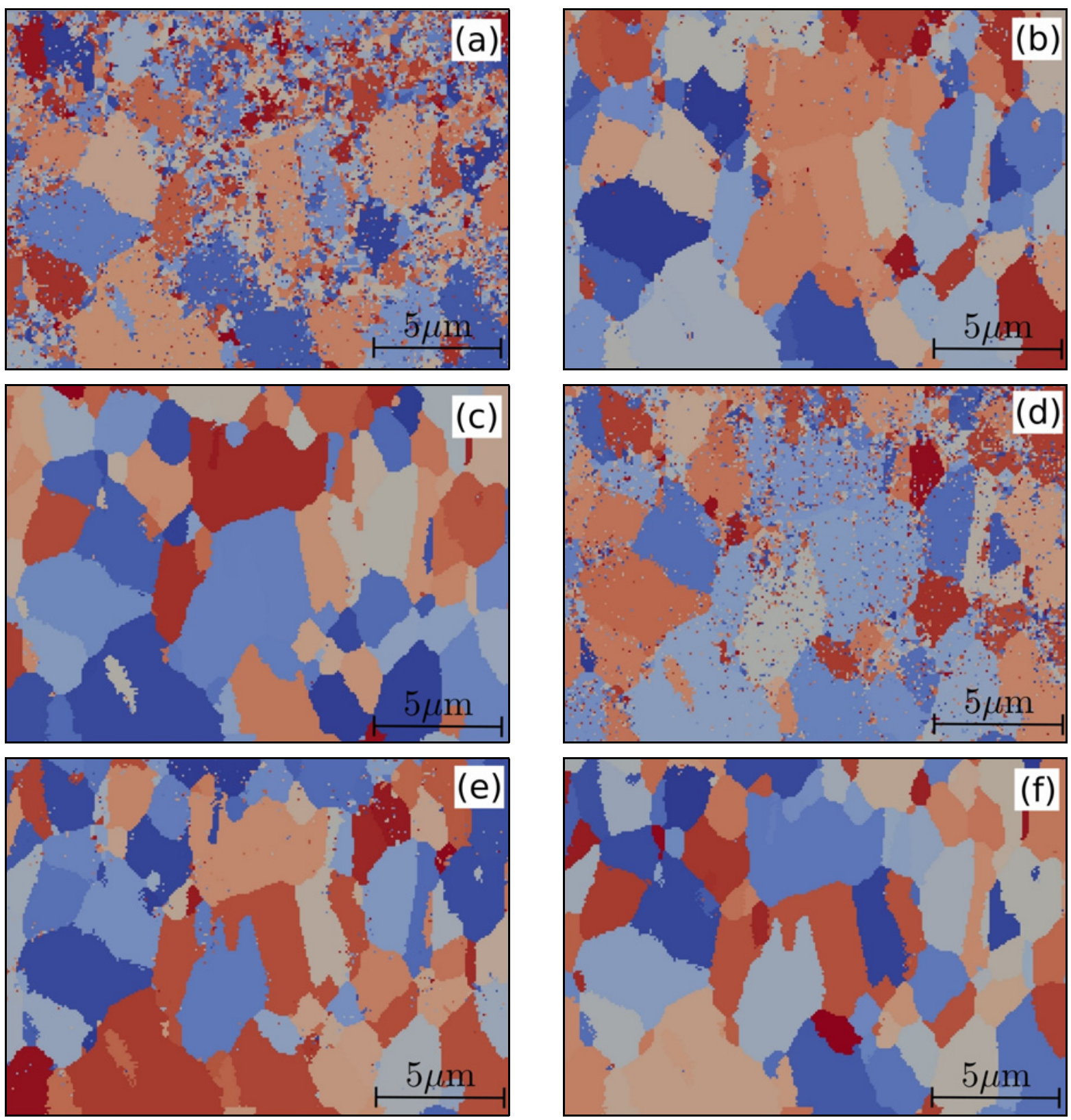

Fig. 14. Pure copper data. The segmentation for the 20th slice in $2 \mathrm{D}$ with the misorientation threshold (a) $0.25^{\circ},(\mathrm{b}) 0.5^{\circ}$, and (c) $5^{\circ}$ and the same section from $3 \mathrm{D}$ reconstruction with the misorientation threshold (d) $0.25^{\circ}$, (e) $0.5^{\circ}$, and (f) $5^{\circ}$. Grains are coloured at random.

grain boundaries with lower misorientation than a given threshold is more important than the threshold itself.

Earlier has been observed that for the AlMgSc alloy, the difference between the segmentation in $2 \mathrm{D}$ and $3 \mathrm{D}$ is significant when the misorientation threshold is chosen so that $10 \%$ of the grain boundaries have lower misorientation than this threshold. We can see the same behaviour in the simulation study in Section 2. Here, for pure $\mathrm{Cu}$, the difference is significant when the misorientation threshold is chosen so that $15 \%$ of the grain boundaries have lower misorientation. This is probably caused by the fact that in the latter case, the $\mathrm{Cu}$ specimen is quite thin, so the $3 \mathrm{D}$ segmentation is closer to 2D segmentation than in the former case (AlMgSc). Nevertheless, it can be supposed that in a wider specimen, the percentage for which we can observe significant differences would be around $10 \%$, too. Just note, that the effect of material thickness on the difference between segmentation in $2 \mathrm{D}$ and $3 \mathrm{D}$ has already been studied in Section 2 .

\section{Five-parameter analysis of grain boundaries}

In the last section, we seemingly get away from 

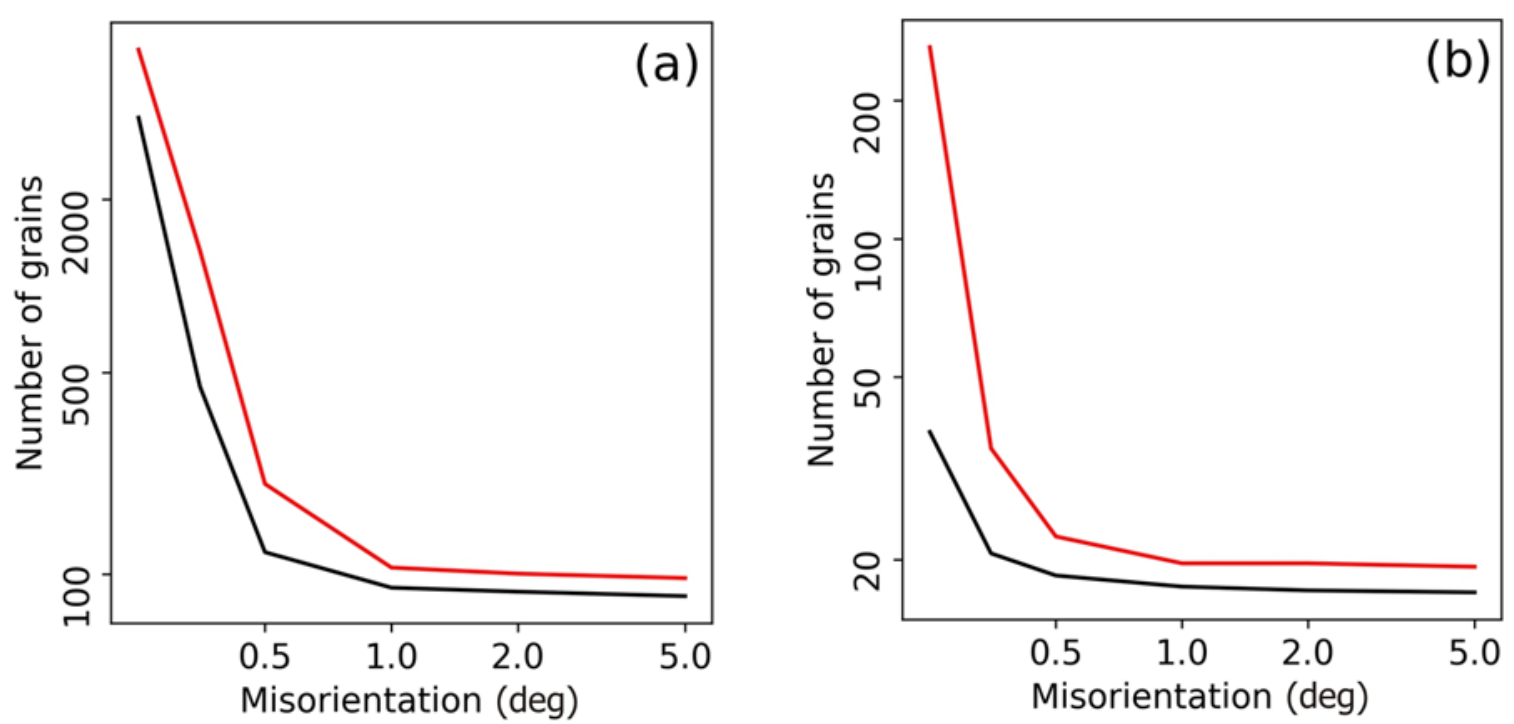

Fig. 15. Pure copper data. Dependence of the mean number of grains in one slice on the misorientation threshold while processing by 2D method (red line) and 3D method (black line). (a) the largest grains which cover in total $90 \%$ of the whole slice and (b) the largest grains which cover in total $50 \%$ of the whole slice.

the main topic of the paper (segmentation) and study the relation between misorientations and orientations of grain boundaries (OGB) in the $\mathrm{Cu}$ specimen from Section 3. According to the literature $[1,20]$, this belongs to the so-called five-parameter analysis, since we employ the angle/axis $(\theta, r)$ of misorientation (three parameters) and two parameters describe the OGB (reflecting the $X Y Z$ coordinate system of the material specimen, $Z$ is the vertical axis). In the first step, the pairs $(\theta, r)$ were used, where $r$ is a $3 \mathrm{D}$ unit vector. For each grain boundary, the Rodrigues vector was $R=\tan \left(\frac{\theta}{2}\right) / r$, obtaining a sample $R_{1}, \ldots, R_{n}$, where $n$ is the total number of registered boundaries.

A representation of the Rodrigues vectors $R=$ $(x, y, z)$ corresponding to various special grain boundaries for a cubic lattice in a $z$-perspective diagram is presented in [1, Fig. 9.19a], and it will serve for the classification. Due to symmetries, it is sufficient to consider vectors with $x \geq y \geq z$, cf. [1, Table 9.2], therefore the diagram is triangular and for points $(x, y)$ close to the origin also the $z$-coordinate and consequently the misorientation angle $\theta$ is small.

In the second step of the five-parameter analysis, we consider OGB represented by unit vectors normal to grain boundaries. Their directions are approximated by a line through centroids of neighbouring grains, which leads to a sample of axial spherical data. The aim is to study whether there is a statistical dependence between OGB and misorientations. For subsamples of special grain boundaries selected using the Rodrigues vector, it will be tested whether OGB are uniformly randomly distributed. An exploratory analysis in terms of eigenvalues of the scatter matrix can be provided [21], in the uniform case, theoretically, all eigenvalues are equal. The formal Gine's test [21] suf-
Ta b l e 3. Cu sample C1 studied under three different segmentations. Uniformity testing of orientations (OGB) in subsamples of $\Sigma 3$ and $\Sigma 9$ grain boundaries. In the columns, the angle is the misorientation threshold in degrees, $n$ is the size of each subsample, $\bar{t}_{1}, \bar{t}_{2}$, and $\bar{t}_{3}$ are the eigenvalues of the scatter matrix in descending order, $G_{n}$ is the value of Gine's test statistic, "in", "out" are numbers of points in the inner, outer area in diagrams in Fig. 19. The critical value for the test on a $5 \%$ confidence level is 2.207 ; thus in all cases, uniformity is rejected.

\begin{tabular}{rrrrrrrrr}
\hline & Angle & $n$ & $\bar{t}_{1}$ & $\bar{t}_{2}$ & $\bar{t}_{3}$ & $G_{n}$ & in & out \\
\hline$\Sigma 3$ & $5^{\circ}$ & 845 & 0.38 & 0.35 & 0.27 & 2.94 & 353 & 492 \\
$\Sigma 9$ & $5^{\circ}$ & 335 & 0.42 & 0.36 & 0.22 & 3.72 & 106 & 229 \\
$\Sigma 3$ & $20^{\circ}$ & 230 & 0.52 & 0.38 & 0.10 & 10.27 & 30 & 200 \\
$\Sigma 9$ & $20^{\circ}$ & 108 & 0.58 & 0.34 & 0.08 & 7.11 & 11 & 97 \\
$\Sigma 3$ & $25^{\circ}$ & 94 & 0.56 & 0.36 & 0.08 & 5.79 & 13 & 81 \\
$\Sigma 9$ & $25^{\circ}$ & 52 & 0.54 & 0.42 & 0.04 & 3.69 & 1 & 51 \\
\hline
\end{tabular}

fers from the fact that OGB's are, strictly speaking, not stochastically independent. When the uniformity is violated, then we have to describe what OGB's are preferred in a given group. To this purpose, we use Lambert's equal-area projection of the hemisphere of OGB onto a circle [21].

For a representative sample (denoted $\mathrm{C} 1$ ) of the $\mathrm{Cu}$ specimen from Section 3.2 with 2265 grains under the misorientation threshold $5^{\circ}$, we obtain a histogram of misorientation angles and a diagram of projected Rodrigues vectors in Figs. 18a,b, respectively. There are two clusters at the hypotenuse of the triangular diagram b). The upper one corresponds to the special $\Sigma 3$ boundaries, while the lower cluster corresponds to the special $\Sigma 9$ boundaries. The Gine's test of uniformity 

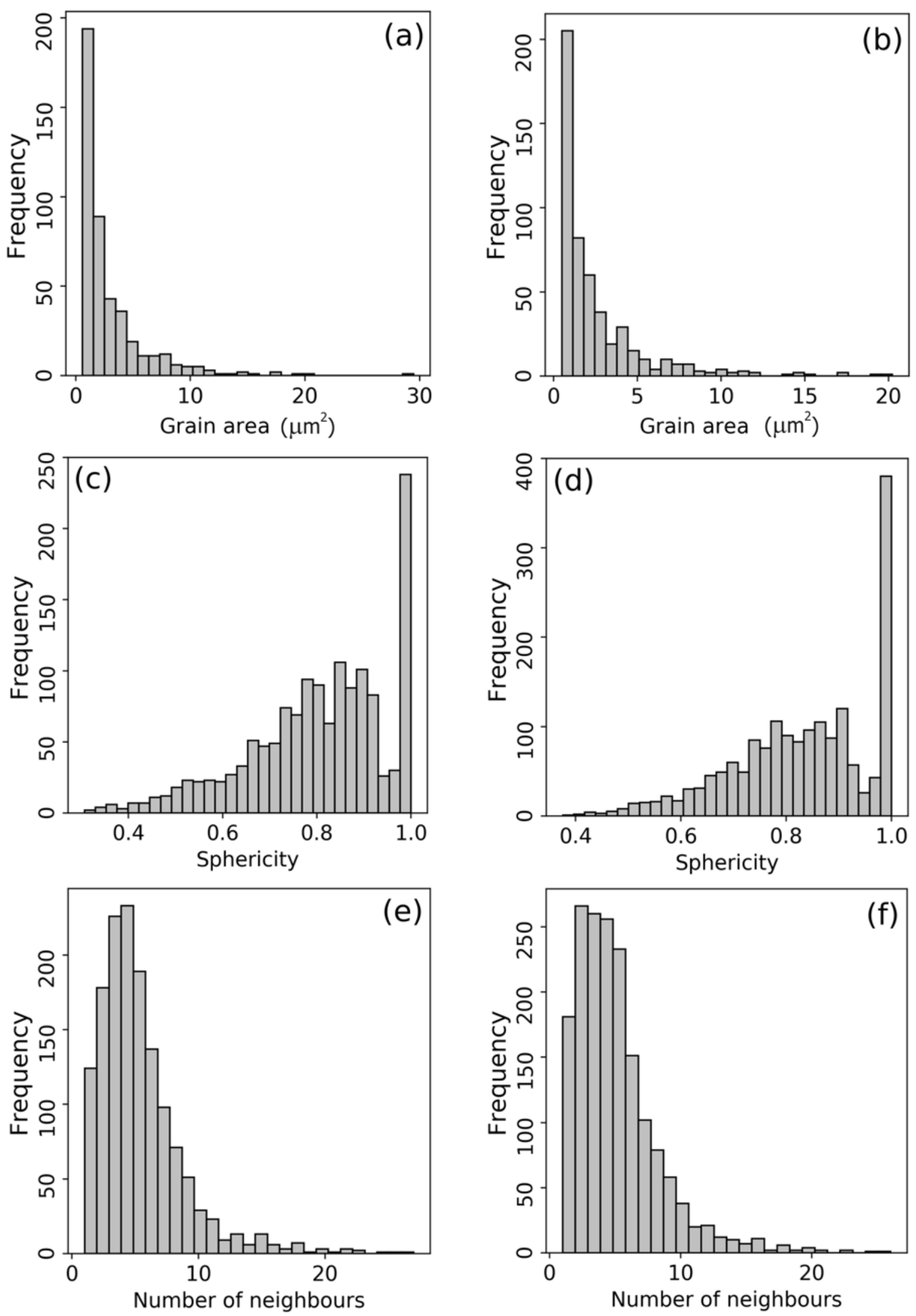

Fig. 16. Pure copper data. Histograms of the area of the largest grains which cover in total $90 \%$ of entire slices: (a) the segmentation in 3D - 988 grains are omitted, (b) the segmentation in 2D - 1217 grains are omitted. Histograms of the sphericity of grains: (c) the segmentation in 3D, and (d) the segmentation in 2D. Histograms of the number of neighbours of grains: (e) the segmentation in $3 \mathrm{D}$ and (f) the segmentation in $2 \mathrm{D}$. The chosen misorientation threshold is $5^{\circ}$. 

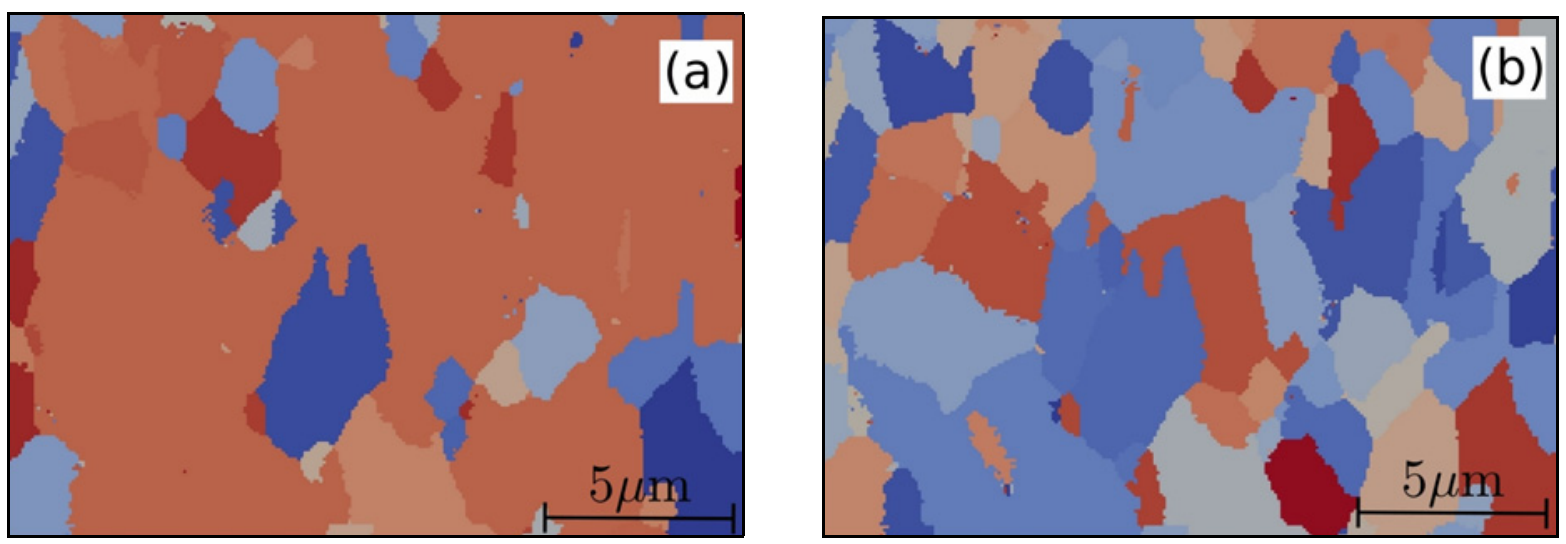

Fig. 17. Pure copper data. Segmentation of a sampled slice (namely slice No. 20) with the misorientation threshold equal to $25^{\circ}$; (a) the segmentation in 3D (79 grains) and (b) segmentation in 2D (161 grains).
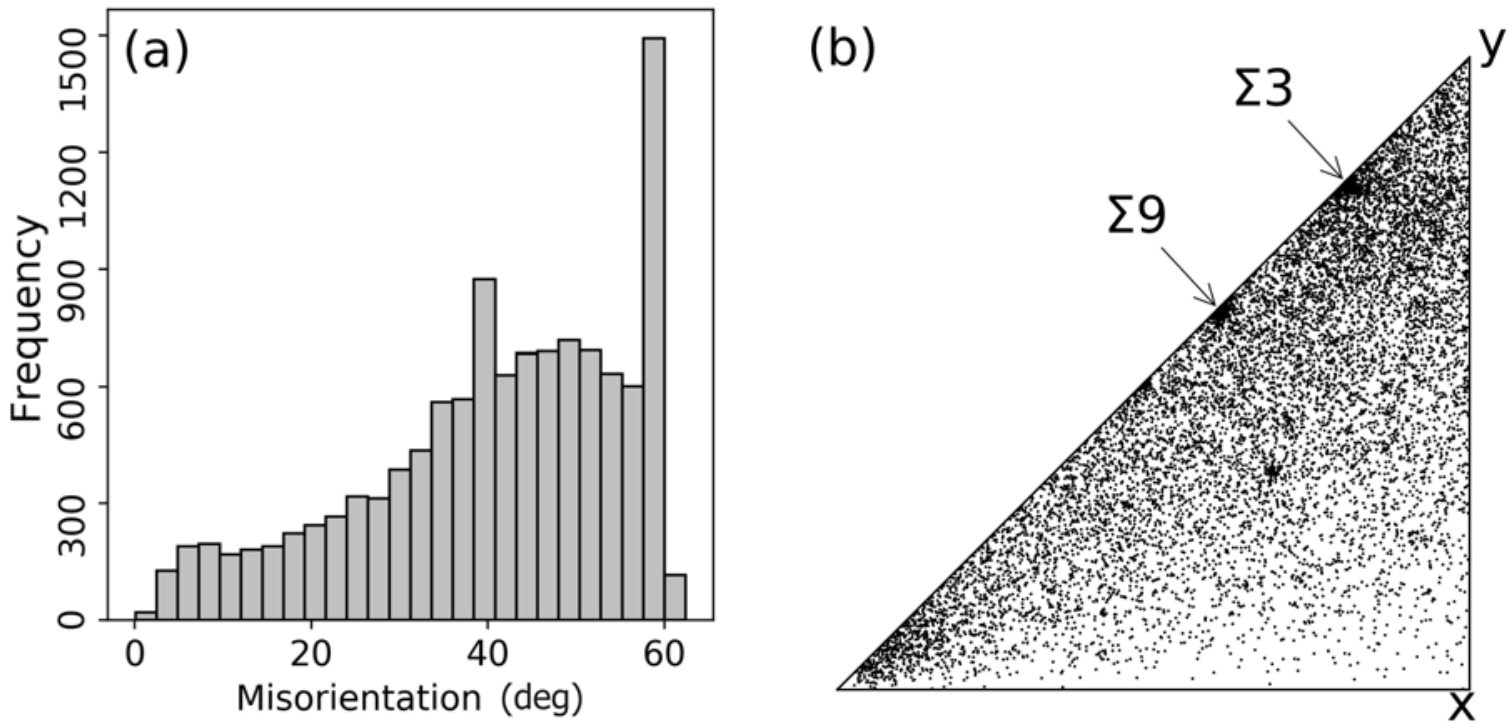

Fig. 18. Cu sample $C 1$ with 11513 grain boundaries. (a) Histogram of misorientation angles of grain boundaries with peaks at $39^{\circ}$ and $60^{\circ}$ which correspond to special boundaries $\Sigma 9$ and $\Sigma 3$. (b) $z$-perspective diagrams of Rodrigues vectors, clusters at the hypotenuse were observed, the darts were seen. The upper one corresponds to the special $\Sigma 3$ boundaries, while the lower cluster corresponds to the special $\Sigma 9$ boundaries.

of OGB for both groups of $\Sigma 3$ and $\Sigma 9$ is rejected on a $5 \%$ confidence level; see the first two lines of Table 3. In the Lambert projection, see Figs. 19a,d, we observe more OGB's outside inner circle of equal area, which means special grain boundaries prefer to form low angles with the $Z$-axis of the specimen. This nonuniformity is more apparent for group $\Sigma 9$ as can also be observed from the eigenvalues of the scatter matrix (the differences between eigenvalues are higher in this case). See the following interpretation of these results.

It was shown [22] that grains in HPT processed materials are not fully equiaxed even after the application of large strains. The grains after HPT are usually elongated in the HPT shear direction. It means that the longest grain axes are more or less parallel to the HPT shear direction in the microstructure after HPT. This structural feature may influence the formation of special grain boundaries. It is generally accepted that the grain boundaries are favoured nucleation sites [23] for twin formation. It may be suggested that special boundaries are predominantly nucleated and grew perpendicularly to the longest grain axis of original elongated grains formed during HPT. For this reason, the faces of special boundaries can appear to be close to parallel with shear direction ( $Z$-axis). This structural feature needs to be studied further.

Finally, we are getting back to the main topic of this paper, that is segmentation, here in $3 \mathrm{D}$. The above described five-parameter analysis has been performed on the same sample with two more misorientation thresholds, namely $20^{\circ}$ and $25^{\circ}$, cf. Fig. 17 . They lead to 4394, 1830 grains, and 1112, 798 grain boundaries, respectively. These thresholds are below the misorientation angle of both $\Sigma 3$ and $\Sigma 9$ special bound- 

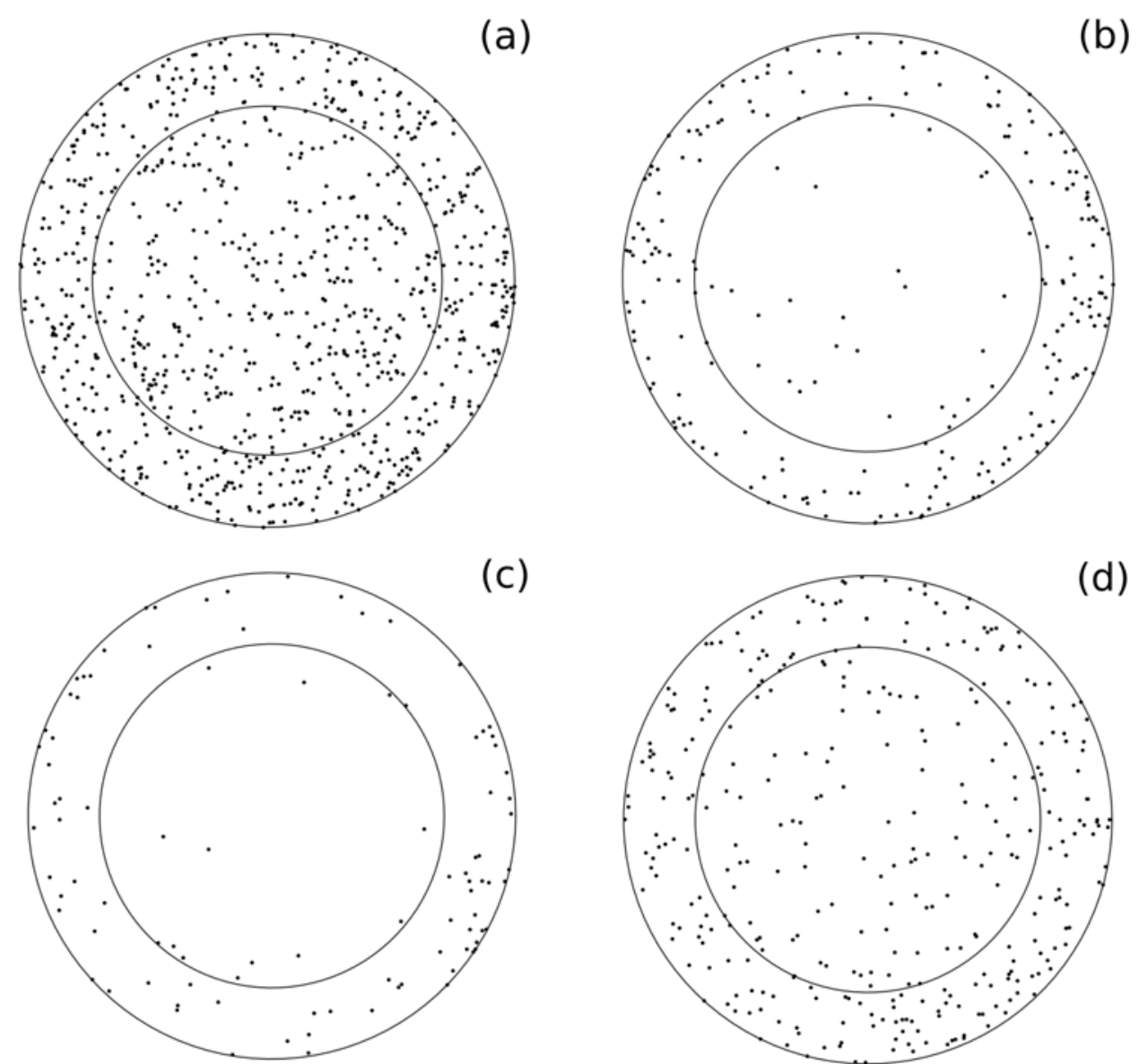

(d)
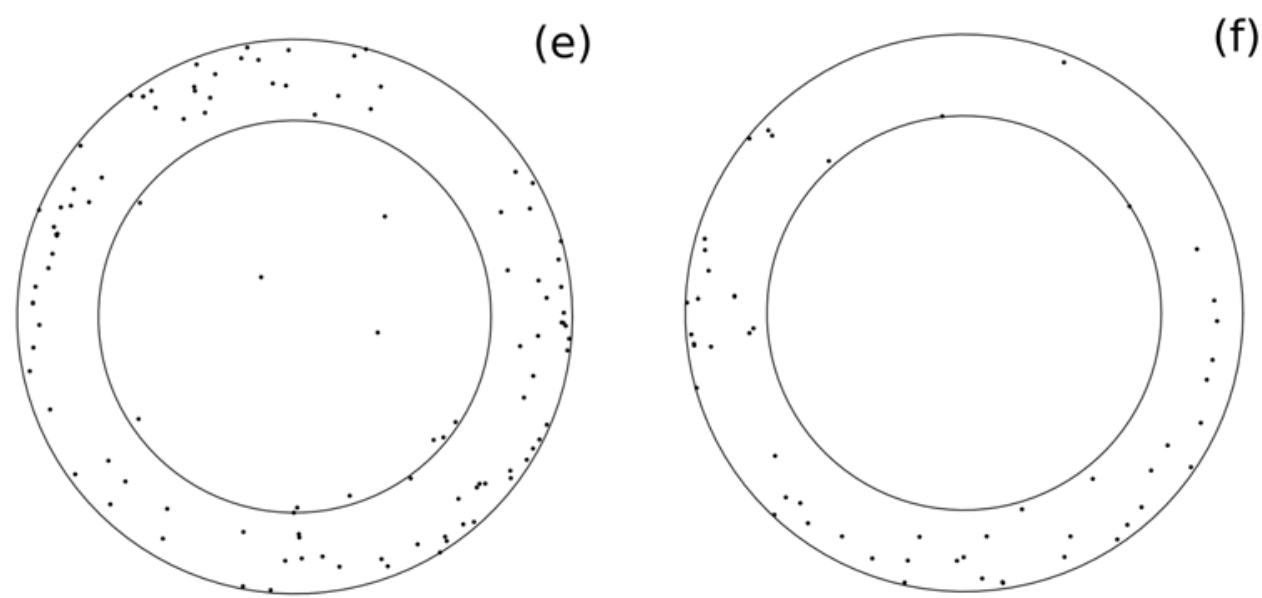

Fig. 19. Equal-area projections of spherical data OGB for special boundaries in $\mathrm{Cu}$ sample $\mathrm{C} 1$ with three levels of misorientation threshold $5^{\circ}, 20^{\circ}$, and $25^{\circ}$. The cases are (a) $\Sigma 3$ boundaries, $5^{\circ}$, (b) $\Sigma 3$ boundaries, $20^{\circ}$, (c) $\Sigma 3$ boundaries, $25^{\circ}$, (d) $\Sigma 9$ boundaries, $5^{\circ}$, (e) $\Sigma 9$ boundaries, $20^{\circ}$, and (f) $\Sigma 9$ boundaries, $25^{\circ}$. In the diagrams, there is an inner circle ("in"), which has the same area as the remaining annulus ("out"). In Table 3, there are numbers of points in both subareas (in, out) that suggest in which way the uniformity of OGB is violated.

aries. In Table 3, there are results of the uniformity testing of OGB in these cases, and in Fig. 19, there are all equal-area projections. There is even more evidence for the rejection of the OGB uniformity hypothesis after these unrealistic thresholds, since the inner circles in Fig. 19 are getting almost vacant. For the misori- entation threshold $25^{\circ}$, there is a large grain G such that some very small grains are entirely contained in $\mathrm{G}$, and their OGB is not well defined. In this noise, our procedure is able to find the right special boundaries with well defined OGB as conjectured from the experiment in the previous paragraph. 


\section{Conclusions}

The segmentation into grains differs when making $2 \mathrm{D}$ and $3 \mathrm{D}$ processing, as was shown both in the simulation study (Section 2) and in the real data analysis (Section 3). It has been found out that the segmentation in $2 \mathrm{D}$ is more sensitive to the noise. This is important, especially for the choice of low misorientation threshold. Furthermore, there appear processes that join more grains into one bigger grain for the same misorientation threshold in the $3 \mathrm{D}$ case than in the $2 \mathrm{D}$ case. This behaviour is important when at least 10 $15 \%$ of the boundaries have the misorientation smaller than the chosen threshold. This effect must be taken into account in the case when working with a specimen where low angle grain boundaries are predominant. For such a specimen, the difference between segmentation in $2 \mathrm{D}$ and $3 \mathrm{D}$ could be significant.

Moreover, we observed that also the thickness of the specimen affects the difference between $2 \mathrm{D}$ and $3 \mathrm{D}$ segmentation. It is not very distinct when the specimen is too thin, because there is not enough space for joining the grains, which causes the difference. On the other hand, we have noticed that the morphology of the grains does not play an essential part in this sense.

The percentual expressions on misorientation thresholds concluded in this paper may be specific observations. Generally, we can say that for the 2D segmentation, the chosen misorientation threshold should be larger than that one for the $3 \mathrm{D}$ segmentation. Moreover, if we work with $3 \mathrm{D}$ data (for example, a specimen from 3D-EBSD), we should respect the distribution of misorientations of boundaries when choosing the misorientation threshold for the segmentation into grains. As an additional result, using three levels of 3D segmentation, we showed and interpreted that orientations of special boundaries in the $\mathrm{Cu}$ specimen are not uniformly distributed.

\section{Acknowledgements}

The financial support of the Czech Science Foundation, project no. 17-00393J is highly appreciated. F. Seitl was also supported by Charles University, project SVV 260580.

\section{References}

[1] O. Engler, V. Randle, Introduction to Texture Analysis. CRC Press, Boca Raton (2010). ISBN 978142006 3653.

[2] A. J. Schwartz, M. Kumar, B. L. Adams, D. P. Field (Eds.), Electron Backscatter Diffraction in Materials Science, 2nd ed., Springer, Boston (2009). ISBN 9780-387-88135-5. doi:10.1007/978-0-387-88136-2

[3] T. L. Burnett, R. Kelley, B. Winiarski, L. Contreras, M. Daly, A. Gholinia, M. G. Burke, P. J. Wither,
Large volume serial section tomography by Xe-Plasma FIB dual beam microscopy, Ultramicroscopy 161 (2016) 119-129. doi:10.1016/j.ultramic.2015.11.001

[4] P. Bobrowski, M. Faryna, K. Głowiński, Evaluation of grain boundary plane distribution in yttria stabilized polycrystalline zirconia based on 3D EBSD analysis, Mater. Charact. 122 (2016) 137-141. doi:10.1016/i.matchar.2016.10.033

[5] H. Pirgazi, K. Głowiński, A. Morawiec, L. A. Kestens, Three-dimensional characterization of grain boundaries in pure nickel by serial sectioning via mechanical polishing, J. Appl. Crystal. 48 (2015) 1672-1678. doi:10.1107/S1600576715017616

[6] P. Bobrowski, M. Faryna, Z. Pędzich, Microstructural characterization of yttria-stabilized zirconia sintered at different temperatures using 3D EBSD, 2D EBSD and stereological calculations, J. Mater. Eng. Perform. 26 (2017) 4681. doi:10.1007/s11665-017-2794-4

[7] A. Okabe, B. Boots, K. Sugihara, S. N. Chiu, Spatial Tessellations: Concepts and Applications of Voronoi Diagrams. Wiley, New York (2000). ISBN 978-0-47198635-5.

[8] K. P. Mingard, M. Steward, M. G. Gee, S. Vespucci, C. Trager-Cowan, Practical application of direct electron detectors to EBSD mapping in 2D and 3D, Ultramicrosc. 184 Part A (2018) 242-251. doi:10.1016/j.ultramic.2017.09.008

[9] Welcome to OIM analysis (1997-2019). EDAX, AMETEK, Inc., Materials Analysis Division.

[10] Y. L. Niu, S. J. Jia, Q. G. Liu, S. Tong, B. Li, Y. Ren, B. Wang, Influence of effective grain size on low temperature toughness of high-strength pipeline steel, Materials 12 (2019) 3672. doi:10.3390/ma12223672

[11] F. C. Lin, A. Godfrey, D. J. Jensen, G. Winther, 3D EBSD characterization of deformation structures in commercial purity aluminum, Mater. Charact. 61 (2010) 1203-1210. doi:10.1016/j.matchar.2010.07.013

[12] J. Kopeček, J. Staněk, S. Habr, F. Seitl, L. Petrich, V. Schmidt, V. Beneš, Analysis of the polycrystalline microstructure of AlMgSc alloy observed by 3D EBSD, Image Anal Stereol 39 (2020) 1-11. doi: $10.5566 /$ ias. 2224

[13] O. Sedivý, T. Brereton, D. Westhoff, L. Polívka, V. Beneš, V. Schmidt, A. Jäger, 3D reconstruction of grains in polycrystalline materials using a tessellation model with curved grain boundaries, Philos. Magaz. 96 (2019) 1926-1949. doi: $10.1080 / 14786435.2016 .1183829$

[14] M. A. Groeber, M. A. Jackson, DREAM.3D: A digital representation environment for the analysis of microstructure in 3D, Integ. Mat. Manuf. Innov. 3 (2014) 5-17. doi:10.1186/2193-9772-3-5

[15] C. Lautensack, S. Zuyev, Random Laguerre tessellations, Adv. Appl. Prob. 40 (2008) 630-650. doi:10.1239/aap/1222868179

[16] F. Seitl, L. Petrich, J. Staněk, C. Krill, V. Schmidt, V. Beneš, Exploration of Gibbs-Laguerre tessellations for three-dimensional stochastic modelling, Method. Comp. Appl. Probab. (2020). doi:10.1007/s11009-019-09757-x

[17] Z. Horita, M. Furukawa, M. Nemoto, A. J. Barnes, T. G. Landgon, Superplastic forming at high strain rates after severe plastic deformation, Acta Mat. 48 (2000) 3633-3640. doi:10.1016/S1359-6454(00)00182-8 
[18] I. Cavarretta, C. O'Sullivan, M. R. Coop, Applying 2D shape analysis techniques to granular materials with 3D particle geometries, AIP Conf. Proc. 1145 (2009) 833-836. doi:10.1063/1.3180057

[19] P. Král, J. Staněk, L. Kunčická, F. Seitl, L. Petrich, V. Schmidt, V. Beneš, V. Sklenička, Microstructure changes in HPT-processed copper occuring at room temperature, Mater. Charact. 151 (2019) 602-611. doi:10.1016/i.matchar.2019.03.046

[20] A. Morawiec, Orientations and Rotations. Computations in Crystallographic Textures. Springer, Berlin (2004). ISBN 978-3-540-40734-8. doi:10.1007/978-3-662-09156-2
[21] K. V. Mardia, P. E. Jupp, Directional Statistics. Wiley, Chichester (2000).

[22] P. Pippan, S. Scheriau, A. Taylor, M. Hafok, A. Hohenwarter, A. Bachmaier, Saturation of fragmentation during severe plastic deformation, Annu. Rev. Mater. Res. 40 (2010) 319-343. doi:10.1146/annurev-matsci-070909-104445

[23] F. J. Humphreys, M. Hatherly, Recrystallization and Relating Annealing Phenomena. Elsevier, Oxford (2004). ISBN 978-0-08-044164-1. doi:10.1016/B978-0-08-044164-1.X5000-2 